$9-21-2021$

Information Avoidance and Celebrity Exposure: The Effect of "Magic" Johnson on AIDS Diagnoses and Mortality in the U.S.

Alexander Cardazzi

Joshua C. Martin

Zachary Rodriguez

Follow this and additional works at: https://researchrepository.wvu.edu/econ_working-papers

Part of the Economics Commons 


\title{
Information Avoidance and Celebrity Exposure: The Effect of "Magic" Johnson on AIDS Diagnoses and Mortality in the U.S.
}

\author{
Alexander Cardazzi ${ }^{1}$, Joshua C. Martin ${ }^{1}$, and Zachary Rodriguez ${ }^{2}$ \\ ${ }^{1}$ West Virginia University \\ ${ }^{2}$ Syracuse University
}

Draft: September 2021

\begin{abstract}
We present evidence that Earvin "Magic" Johnson's announcement that he contracted HIV served as a public-health catalyst for rapidly correcting the public's understanding of who was at risk of infection. Using a novel identification strategy, we present evidence that there was a large but temporary increase in the number of AIDS diagnoses for heterosexual men following the announcement. This effect was concentrated in areas with greater prior exposure to Johnson. We show that these men were both more likely to have been diagnosed via a formal blood test and less likely to die within one decade of their initial diagnosis - suggesting that Johnson's announcement caused an intertemporal substitution in testing which prolonged patients' lifespans as a result of earlier access to medical care. We estimate that Johnson's announcement caused approximately 800 additional men to discover their underlying AIDS diagnosis and, of whom, were more likely to live at least one decade beyond their initial diagnosis date.
\end{abstract}

I think sometimes we think, well, only gay people can get it - "It's not going to happen to me." And here I am saying that it can happen to anybody, even me.

- Earvin "Magic" Johnson

\section{Introduction}

The AIDS pandemic is one of the largest public health crises in the $20^{\text {th }}$ century. While there is typically a large degree of informational asymmetries between health experts and at-risk populations which exacerbate similar contagions, the AIDS pandemic is in many ways defined by it. As the virus became increasingly prevalent throughout the world, scientific understandings of its origins, causes, and consequences rapidly increased, while the general public's understanding lagged behind. Though a substantial majority of Americans during the height of the AIDS pandemic understood how HIV could be contracted, there existed large gaps in knowledge regarding its transmission risks - particularly amongst heterosexuals (Herek and Capitanio, 1998; Herek et al., 2002, 2005). We contend that this behavior is representative of what the Economics literature refers to as information avoidance, wherein biased beliefs directly enter agents' utility functions and create an incentive to avoid information even in situations where it is both useful and low-cost (Golman et al., 2017). 
There are few, if any, information shocks during the AIDS pandemic which were greater than when Earvin "Magic" Johnson publicly announced that he had contracted HIV on November 7, 1991. In this study, we present evidence that Johnson served as a catalyst in the United States for rapidly changing the perception of who was at risk for contracting HIV. We document the effects of Johnson's announcement in both public knowledge of, and testing for, AIDS through the use of data from the the National Health Interview Survey (NHIS). We document a modest $10 \%$ increase in the expectation of being tested in the future for those who had previously been tested for HIV/AIDS. However, for those who had not yet been tested, we document a $40 \%$ in expectation of getting tested.

We compliment this finding by analyzing the AIDS Public Information Data Set (APIDS) from the Centers for Disease Control and Prevention (CDC) which provides individual-level data for AIDS diagnoses at a monthly interval across all major metropolitan statistical areas (MSAs) in the United States. Crucially, the data also contains information regarding the age, gender, race, and transmission category of those diagnosed, which allows us to estimate the effect of Johnson's announcement on groups of individuals with potentially very different prior beliefs regarding their probability of contracting HIV. Specifically, due to the prevalence and extreme stigmatization of AIDS in the gay community at the time, we posit that homosexual individuals were much more likely to be aware of their chances of contracting HIV relative heterosexual individuals at the time of Johnson's announcement. We therefore expect the effect of the announcement to be predominantly concentrated among heterosexuals.

Our empirical analysis employs a difference-in-differences method where homosexual men serve as the control group and heterosexual men are the treated. Using Johnson's announcement as an exogenous shock to popular knowledge and perceptions of HIV, we demonstrate that there was a large, but temporary increase in the number of AIDS diagnoses amongst heterosexual men immediately following the announcement. Given the high stature of Johnson as a professional basketball player at the time, we rely on a variable indicating whether there is a National Basketball Association (NBA) franchise located within the MSA of one's diagnosis in order to proxy for pre-announcement exposure to Johnson as an athlete. This differentiation reveals that exposure to Johnson before his announcement acted as an important explanatory variable in describing differences in diagnoses between groups. Our results indicate that approximately 800 more heterosexual men were definitively diagnosed with AIDS in MSAs with NBA franchises relative to our control group of homosexual men following Johnson's announcement.

We conclude our analysis by estimating the mortality of patients diagnosed with AIDS following Johnson's announcement to rule out alternate mechanisms responsible for the observed increases in AIDS diagnoses. Our results find that heterosexual men in MSAs with NBA franchises were 7\% more likely to live until 2000, relative to heterosexual men in MSAs without NBA franchises. This finding suggests that the results are being driven by an intertemporal substitution in testing behavior rather than behavioral factors such as moral hazard, or a coincidental increase in the prevalence of AIDS within these communities at the time. We also find evidence of heterogeneous effects by race, as the overall result is driven by black and Hispanic men.

These results broadly align with the findings of other researcher such as Godlonton and Thornton (2013) who find that learning of the negative results of a friend or neighbor's HIV test causes a reduction in the perception of one's own risk for contracting HIV. One contribution of this study is to document the other side of this effect - that learning that learning of the positive HIV status of someone who is liked and admired increases one's own perception of risk for contracting HIV. 
The fact that the estimates in our results are driven by individuals residing within MSAs with NBA franchises suggest that familiarity with Johnson played a crucial role in changing individual perception of risks and testing behavior. In doing so, we identify a powerful public health mechanism by linking our results to findings in the literature on information avoidance and how celebrities can influence public risk perception (Oster et al., 2013; Cram et al., 2003). As communities work to fight against the spread of contagious viruses and diseases, our results highlight the importance of both public health messaging and the degree to which influential individuals can ameliorate misinformation and perceptions of risk.

In Section 2, we briefly survey the literature on information avoidance, describing where our analysis fits within the empirical literature on this behavior. We describe the setting of our study in Section 3. In Section 4, we outline our identification strategy and guiding hypotheses. Section 5 details the data used in our study, and Section 6 describes the empirical strategy we use to analyze Johnson's effect on AIDS diagnoses and mortality. We analyze the data in Section 7 establishing meaningful treatment effects for both diagnoses and mortality, as well as, identifying heterogeneous effects within our sample. In section 8 , we provide concluding discussion.

\section{Literature Review}

\subsection{Health Consequences of Information Avoidance}

Information avoidance can have both serious private and external costs for those involved. Often the sooner that one seeks treatment for an illness, the more quickly that treatment can begin. Yet the fear of receiving negative news pertaining to one's health can adversely affect individual's willingness to seek out information regarding their vulnerability to, or probability of transmitting, any illness. Golman et al. (2017) formalized the term "information avoidance" in the Economics literature by analyzing this type of behavior through the lens of rational-agent decision making.

Economists typically assume that information is valuable as a tool for reducing uncertainty in decision making processes. As a result, forward looking, utility maximizing agents will purchase and consume information up until the point where its marginal benefit equals its marginal cost. The novel addition of economic theorists was to build models of decision making wherein it is optimal to avoid seeking out information when biased beliefs enter the agent's utility function - even if that information is useful, free, and independent of strategic considerations.

This impact can particularly be seen in contexts with high negative externalities. The degree to which the costs of information avoidance are borne by others is a function of the transmissibility of the action in question. However, trasnmissibility in environments of high stigma can actually increase rather than decrease information avoidance behavior. This can lead to a negative feedback loop through which information avoidance begets contagion, and so on.

Personal health is one major area where the literature identifies information avoidance behavior. Köszegi (2003) provides related theoretical support for this concept by presenting a model where patient's anxious beliefs about the state of their health in the next period enter their utility function. The model allows patients to endogenously chose how the information they gather will affect their beliefs and, thus, utility. If the patient is risk averse, then they will exchange the anxiety of the bad news for the decision making value 
of knowing the state of their health. ${ }^{1}$ As a result, the model predicts that patients can refuse useful, low cost information should their anxiety be sufficiently high.

There has been much empirical research which lends support to this behavioral theory. Oster et al. (2013) find that large amounts of individuals who are at known risk of carrying Huntington's disease (a hereditary degenerative disease which can dramatically limit quality of life and life expectancy) fail to receive relatively inexpensive testing and dramatically underestimate their probability of carrying the disease - even in situations where the information would be highly useful. Additionally, Ganguly and Tasoff (2017) show that individuals, when given the option to avoid paying to be tested for herpes viruses, are willing to pay significantly more in order to avoid being tested for the most severe strain.

We posit that false beliefs led groups of individuals, many of whom purposefully distanced themselves from the gay community, to systematically underestimate their own probability of contracting or transmitting the disease. Beliefs and attitudes toward HIV within the United States at the time also reflected a strong bias toward people living with AIDS (PWAs) which further exacerbated one's unwillingness to get tested. For example, Herek et al. (2002) report that in 1991 one-in-three Americans said that they feared people living with HIV/AIDS, and that one-in-four admitted to feelings of anger or disgust towards them.

Unfortunately, these misconceptions affected more than prejudice towards PWAs or overly cautious behavior in those without the virus. Of those in already at-risk communities, Stokes and Peterson (1998) finds that psychological distress as a result of community stigmatization is a likely indicator for even less safe behavior. For those in historically safer communities, Valdiserri (2002) argues that the high costs of HIV diagnoses, be they tangible costs such as diagnostic or healthcare service costs, or non-tangible costs such as time, lifestyle changes, stigma or expectations of a loss of job, serve as a strong disincentive for seeking HIV testing.

\subsection{Celebrity Exposure and Health Behavior}

Incentivizing individuals to seek out their current health status can be very difficult as a result of psychological tendencies to avoid unpleasant information. However, research has identified educational campaigns and information shocks from celebrities as a potential mechanism for lessening the harm done as a result of information avoidance. For instance, recent studies have found that celebrities can affect discrimination (Alrababah et al., 2019), election outcomes (Garthwaite and Moore, 2013; Wang, 2021), book sales (Garthwaite, 2014), and tax delinquency (Garz and Pagels, 2018). ${ }^{2}$ As these studies show how celebrities can affect public consumption, beliefs, and attitudes, a growing literature identifies how celebrities can quickly educate individuals on potential health risks.

For instance, Nattinger et al. (1998), Cram et al. (2003) and Kelly et al. (1992) find that celebrity endorsements can inform personal health decisions. Nattinger et al. (1998) estimates the effect of Nancy Reagan's mastectomy on the choice of surgery for breast cancer in American women. They find that women who resemble Reagan demographically are less likely to choose breast-conserving surgery, which is an alternative to a mastectomy. Also, Cram et al. (2003) measures the "Katie Couric Effect", which refers to how the newscaster used her celebrity to campaign for colon cancer screenings. Their results show an increase in screenings among women after Couric's campaign. Kelly et al. (1992) demonstrate that there were system-

\footnotetext{
${ }^{1}$ The only assumption for risk adverse agent in this context is one who derives more disutility from bad news than they would gain from an equal amount of good news.

${ }^{2} \mathrm{~A}$ related advancement of this literature studies the effects of exposure to gender (Beaman et al., 2009) and racial minorities (Boisjoly et al., 2006).
} 
atic reductions in risky sexual behavior amongst gay men when respected community leaders held education campaigns on HIV transmission risks. Each study provides strong evidence that people feel more comfortable seeking out information once a demographically representative public figure is also seen as vulnerable to illness.

Relatedly, several studies have qualitatively analyzed the effect of Magic Johnson's public HIV announcement on beliefs and attitudes toward both HIV and PWAs. Pollock III (1994) uses survey evidence collected in Florida directly before and after Johnson's announcement to show a shift in opinion from heterosexuals through increased support for the amount of money dedicated towards AIDS treatment and research. Importantly, this shift in opinion did not exist for homosexuals. Herek and Capitanio (1997) study the relationship between AIDS-related stigma and vicarious personal contact with a public figure with HIV. In doing so they find that Johnson's announcement had the largest impact among those who previously expressed high levels of stigma. Casey et al. (2003) review several small studies further investigating Johnson's effect on health-related attitudes, beliefs, and behavior. They find changes in the number of people being tested after the announcement, beliefs regarding their vulnerability of contracting HIV, and willingness to obtain more information about HIV and AIDS. Last, in studying the responsiveness to public health AIDS messaging among groups of at-risk African American men, Flora et al. (1996) find that Johnson was preferred to other famous black athletes and celebrities (e.g. Michael Jordan, Eddie Murphy, Prince, Bill Cosby, Arsenio Hall, Jesse Jackson).

\section{Setting}

Deaths as a result of AIDS, or acquired immunodeficiency syndrome, were first noticed in the United States within clusters of intravenous drug users and homosexual men in 1981. This sparked the beginning of the idea that the new contagion was a "gay cancer". Due to the population of patients experiencing these symptoms, coupled with widespread prejudice towards them at the time, the general media coined the germ "GRID" or gay-related immune deficiency. Even scientific agencies such the CDC initially labeled AIDS as a " $4 \mathrm{H}$ " disease referring to four of the most at-risk patient types - homosexuals, heroin users, hemophiliacs and Haitians.

While institutions such as the CDC and the general media eventually chose more objective and publichealth conducive descriptors for AIDS, the lasting-impact of their initial mistakes remained. This perception emboldened some who interpreted the pandemic as confirmation of long-held religious beliefs which regarded homosexuality as immoral, but the naive beliefs of the general American public regarding the causes and consequences of the AIDS pandemic was much more widespread. ${ }^{3}$ For example, Herek et al. (2002) shows that at the height of the pandemic $50 \%$ of Americans believed that one could contract HIV either by being coughed/sneezed on by, or through sharing a drink out of the same glass as someone with AIDS. Many Americans during the AIDS pandemic exclusively associated it with homosexual men despite the well-documented risks of acquiring HIV among heterosexuals (Peterman et al., 1988; Padian et al., 1990).

\footnotetext{
${ }^{3}$ In 1993 then world-famous televangelist Billy Graham rhetorically asked an audience, "Is AIDS a judgment of God?... I could not say for sure, but I think so." (Petro, 2015).
} 


\subsection{Magic Johnson and the NBA}

At the start of the 1980s, the rapidly growing NBA was dominated by the Los Angeles Lakers and the Boston Celtics. Magic Johnson and Larry Bird were the stars of each team respectively - seeing both quickly became some of the most famous athletes in the country regardless of sport. ${ }^{4}$ Over the course of his career from 1979 - 1991, Johnson was a first overall pick in the NBA draft, a 5x NBA champion, a 3x NBA finals MVP, a 3x NBA MVP, a 12x All-Star and 9x All-NBA First Team member. Johnson, with the help of Bird and later Michael Jordan, helped to considerably improve the image and direction of the league. However, even with the increasing popularity of the NBA, many games were still either played on a regional television station or were not broadcast at all. Given the nature of regional television contracts from the 1980s and 1990s in addition to the fact that many NCAA or NBA basketball games could occur at the same time, television providers would almost always broadcast the games which occurred geographically closest to the consumer. ${ }^{5}$

On November 7, 1991, Johnson suddenly and unexpectedly announced that he had contracted HIV. He immediately retired from the NBA. This occurred not only at the height of his professional career and fame, but also the peak of the AIDS pandemic within the United States. In the history of the AIDS pandemic, there are few examples of shocks to public opinion or knowledge greater that of Earvin "Magic" Johnson. Widely considered to be one of the greatest basketball players of all time, Johnson was not only the face of the fastest growing sport in the United States, the NBA, but of all American sports. Perhaps most importantly for this study, Johnson represented a widely adored, non-injection-drug using, heterosexual male. ${ }^{6}$

\subsection{Changing attitudes towards AIDS}

The news that Magic Johnson had contracted HIV was instant national news and became a front-page headline across the United States. ${ }^{7}$ We demonstrate the importance of Johnson's announcement using data from the 1991 National Health Interview Survey (NHIS). ${ }^{8}$ This survey gives us the ability to identify sudden changes in knowledge and attitudes resulting from Johnson's announcement. We test for increases in knowledge about AIDS in Figure A.11. This figure reveals that there was a 5-10 percentage point increases in the percentage of respondents who reported having heard about HIV/AIDS via television, radio or print media in the week immediately after Johnson's announcement.

Table 1 continues this investigation by revealing large, sudden increases in individual's understandings of AIDS. Specifically, we find evidence that respondents show increases in understanding that one can have AIDS but still feel healthy and that one can have HIV without having yet developed AIDS. Further, this table shows that there was an increased familiarity with AZT (an FDA-approved drug which delayed the speed with which HIV developed into AIDS) and decrease in the number of those who had not yet been tested due to their perception that they were not at risk.

We also analyze questions about respondent's beliefs of the probability that they either had, or that

\footnotetext{
${ }^{4}$ His fame and recognition persists to present day. Johnson was recently ranked as the $5^{\text {th }}$ best basketball player of all time according a 2020 list on the subject done by ESPN.com.

${ }^{5}$ In Appendix Figure A.5, we also demonstrate that the NBA is more popular in places where an NBA team exists compared to similar places without an NBA team while sports in general are not.

${ }^{6}$ While it was widely speculated that Johnson had acquired HIV via homosexual sex, this myth was quickly debunked as national attention rapidly shifted to whether Johnson had unknowingly infected his then pregnant wife.

${ }^{7}$ Figure A.12 displays the daily number of mentions of Johnson's name in print newspaper sources - peaking at nearly four times the number of average mentions as he had typically received.

${ }^{8}$ NHIS is a cross-sectional household interview survey, which contains approximately 35,000 households and 87,500 individuals. These data is used by several government agencies in order to track the progress of toward achieving national health objectives.
} 
Table 1: NHIS Questions Relevant to AIDS Knowledge

\begin{tabular}{|c|c|c|c|}
\hline Survey Question & Pre-Magic & Post-Magic & $\mathrm{p}$-value \\
\hline Have AIDS, Feel Healthy & 0.909 & 0.932 & $9.11 \mathrm{e}-11$ \\
\hline Have Virus, Not AIDS & 0.922 & 0.952 & $1.76 \mathrm{e}-21$ \\
\hline Heard of AZT & 0.519 & 0.577 & $2.44 \mathrm{e}-17$ \\
\hline No Test b/c No Risk & 0.913 & 0.897 & $9.56 \mathrm{e}-04$ \\
\hline Chance of Having AIDS $^{+}$ & 0.263 & 0.295 & 0.043 \\
\hline Chance of Getting AIDS ${ }^{+}$ & 0.357 & 0.384 & 0.106 \\
\hline Chance of Having AIDS $^{-}$ & 0.178 & 0.209 & $1.34 \mathrm{e}-06$ \\
\hline Chance of Getting AIDS ${ }^{-}$ & 0.250 & 0.270 & $2.79 \mathrm{e}-03$ \\
\hline
\end{tabular}

Note: Calculations take the difference in mean response percentage for each question from week 1-44 to that from weeks 45-52 in 1991. The first half of this table displays the percentage of 1991 NHIS respondents who answered affirmatively to the question asked. The second half reports the percent of individuals who did not answer "none" for their chance of having/getting AIDS. In addition, $\mathrm{a}+(-)$ mark denotes the subset of respondents who had (not) been tested for HIV at least once before.

they eventually would, develop AIDS. The bottom half of Table 1 reports these results according to whether the respondent had previously been tested for HIV/AIDS. We use this variable as a proxy to differentiate between groups of respondents with plausibly significantly different risk perceptions. Respondents were allowed a categorical answer of either high, medium, low, or none. ${ }^{9}$ We code these responses as being equal to zero if the respondent reported "none", and equal to one otherwise. We observe similar increases in the levels for all four groups, but the percent increases of those who viewed themselves as lower risk were significantly greater.

The survey also includes questions regarding the expectations of being tested in the future. Respondents were asked if they expected to have an AIDS test in the next twelve months. In Figure 1, we show the differences in stated expectations given previous testing history. We find a modest increase $(\approx 10 \%)$ in the expectation of being tested in the future for those who have previously been tested following Johnson's announcement. However, for those who had never been tested, we see a much larger increase $(\approx 40 \%)$. This evidence, in combination with earlier results in Table 1 , suggests that those who were less familiar with their risk level updated their future expectations significantly more than those who were more familiar. ${ }^{10}$

We compare the magnitude of these effects to a similar information shock in the history of the AIDS pandemic in the United States: Ryan White. An otherwise ordinary American teenager living in rural Indiana, White was a hemophiliac who contracted HIV via a tainted blood transfusion. Unlike the thousands of others of hemophiliacs with HIV like him, White's story gained national attention due to groups of teachers and parents who rallied to prevent him from returning to school - despite the medical consensus at the time which was that his risk of transmission to others was minuscule. The lengthy legal appeal process, repeated death treats to both he and his family, and the fact that he was viewed as "innocent" (compared to gay men who were viewed as "guilty"), lead White to become the face for AIDS research advocacy and public education in the late 1980s and early 90s. We find that neither the week of the initial nationwide reporting of the White story nor the week of Congress's passage of the public health act in his name had nearly as large of an impact as Johnson. ${ }^{11}$

\footnotetext{
${ }^{9} 15 \%(78.5 \%)$ of all survey respondents reported having (not) had an HIV/AIDS test in the past. $93.4 \%(\mathrm{~N}=42,725)$ of respondents answered this question.

${ }^{10}$ However, while there is a significantly larger change in those who have never been tested, it is still the case that those who had been tested before were much more likely to be tested in the future.

${ }^{11}$ Recent research has identified the 1992 presidential election as a critical moment in the perception of homosexuals as
} 
Figure 1: Percent of Respondents Expecting to be Tested for AIDS in the Next Year

Previously Had AIDS Test

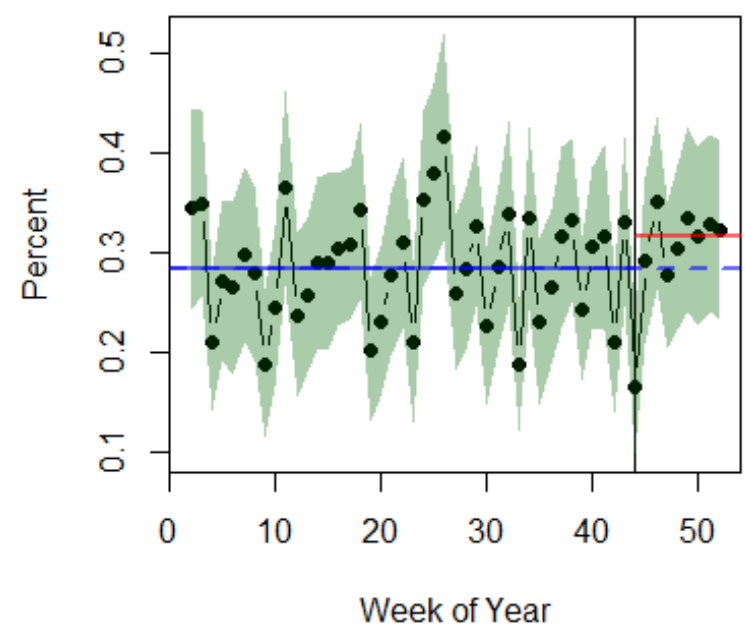

Never Had AIDS Test

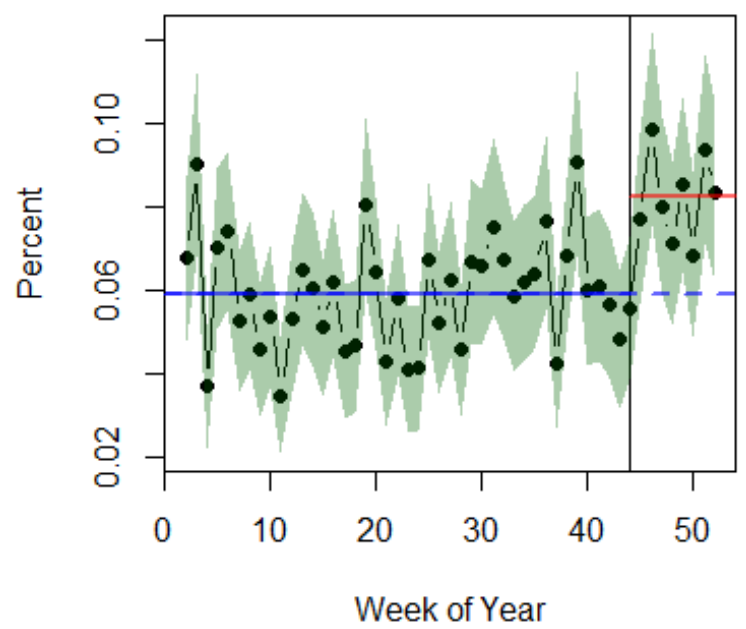

Note: Figure represents respondents from the 1991 NHIS survey being asked if they expect to be tested for HIV/AIDS in the next year. The left (right) panel is a subset of individuals who had (not) been tested for the virus before.

While these results provide strong evidence that Johnson's announcement acted as an information shock to people's beliefs about their own vulnerability to HIV, there are important limitations in using these data. First, there is neither enough demographic nor geographic information known about the respondents of these surveys to account for the large heterogeneity in the prevalence of HIV across different regions, states cities or groups in the United States. Failure to account for these differences at the time of the announcement undermines our ability to make claims about changes in risk preferences. Relatedly, whether a respondent has been tested only weakly proxies for underlying beliefs. This is made even more true if a sufficiently high number of those sampled came from abnormally high or low risk areas. Finally, a representative sample of the American population likely under-represents individuals who were most at risk of infection in 1991, where Johnson's impact would be greatest and matter most.

\section{Identification \& Hypotheses}

As seen in our analysis in Section 3.2, Johnson's announcement acted as a catalyst for changing both public opinion and knowledge of the virus. This effect represents the opposite side of the mechanism discovered by Godlonton and Thornton (2013). Albeit in a significantly different setting, they demonstrate that individuals engage in riskier sexual behavior after learning of a peer's negative HIV/AIDS test.

In order to identify the effect of Johnson's announcement, we consider two possible dimensions along

the AIDS crisis became a critical campaign position for both candidates - demonstrating that there was a sizable change in public opinion regarding moral attitudes towards homosexuality following the election, and that this effect was concentrated in areas which were more impacted by HIV (Fernández et al., 2019; Fernández and Parsa, 2021). In contrast with Johnson's announcment, Figure A.1 shows that there was no significant increase in AIDS diagnoses before or after the election. 
which his impact varied. First, given both the widespread false belief that HIV was a gay cancer and the degree to which homosexuals had disproportionately been affected by it, we posit that there were significant differences in the initial levels of risk perception between homosexual and heterosexual individuals in 1991. Further, although Johnson was an unquestionable superstar at the time, the response to his diagnosis likely also varied according to one's level of familiarity, or even similarity, with him. Unlike today, the NBA was routinely on tape delay and were only consistently broadcast within the immediate viewing area of the home team. Thus, typical individuals residing within MSAs with an NBA franchises were likely to be significantly more familiar with Johnson prior to his announcement.

In order to test for the mechanism familiarity, we compare the differences in homosexual and heterosexual AIDS-related outcomes in MSAs with an NBA franchise to those without one. We expect the effect of Johnson's announcement to concentrate in these MSAs, as a baseline familiarity with the individual or celebrity who provides personal health information has been shown to stimulate people into inquiring about their health risks (Cram et al., 2003; Metcalfe et al., 2011; Evans et al., 2014). In testing for the mechanism of similarities, we compare AIDS-related outcomes across racial groups. We hypothesize that Johnson, an admired, successful and prominent black celebrity, will have the largest impacts on black and other minority men.

We suspect that this effect will manifest itself in a few observable ways. First, this information shock should have led to an sudden increase in testing for AIDS amongst heterosexuals relative to homosexuals. ${ }^{12}$ In turn, we suspect that this testing shock uncovered a considerable number of previously undiagnosed AIDS cases. Nevertheless, there may be a number of other factors which coincide with an increase in AIDS that are not caused directly by the aforementioned mechanism. For instance, an increase in AIDS diagnoses may simply be due to an increase in the prevalence of the disease or by induced moral hazard. However, HIV infections can go undiagnosed well into their progression into AIDS which allow us to rule out alternate arguments. AIDS diagnoses are necessarily preceded by HIV infections, which are primarily symptomatic only in the first $2-4$ weeks. These symptoms commonly manifest as flu-like and thus are commonly misdiagnosed (Choudhary et al., 2007; Hatano et al., 2013; Group, 2015; Sax et al., 2019). This effect is compounded for heterosexual men whom doctors commonly did not assume were at risk of HIV infection early on in the pandemic. Additionally, though there is considerable variation, individuals can often live unhindered for 3-10 years following their initial infection (Ewings et al., 2008). Given that AIDS depletes the immune system, PWAs often live normal lives absent exposure to any opportune infections. ${ }^{13}$

If the announcement spurred these individuals to receive their diagnosis earlier than they would have otherwise, there are two necessary logical consequences that would follow. First, an increase in diagnoses should be concentrated amongst asymptomatic individuals whose disease is relatively less progressed. Thus, those who are tested, and therefore diagnosed, will require formal blood work to confirm or reject their HIV/AIDS status. Second, these individuals diagnosed earlier will have greater access to medical advice and treatment. At this stage in the crisis, medical treatment was the most important factor in slowing the disease. Therefore, when individuals are diagnosed at earlier stages of the disease, they are often able to extend their expected lifespan, or prognosis.

\footnotetext{
${ }^{12}$ Alpert et al. (2018) use a similar identification strategy, but in the context of a nation-wide reformulation of OxyContin and its effect on heroin abuse in the U.S. In this study, the "treatment" is denoted by each state's pre-reformulation level of opioid abuse. Therefore, states with relatively less abuse act as a control group. For our study, we take a similar approach in that we analyze differences in groups who have varying beliefs and knowledge of the risks of AIDS, using groups with stronger prior beliefs and knowledge of AIDS as our control group.

${ }^{13}$ See the Appendix section A.2 for more discussion on this matter.
} 


\section{Data}

Data for the main analyses come from multiple sources. We obtain individual AIDS diagnoses from 1982 to 2002 via the CDC's AIDS Public Information Data Set (APIDS). We augment these data with death records from the National Center for Heath Statistics, American Community Survey population estimates, and a Homosexuality Index from Walther and Poston Jr (2004). We describe each of these sources in detail in this section.

\subsection{AIDS Public Information Data Set}

The CDC began tracking individual AIDS diagnoses across metropolitan areas with populations of more than 500,000 persons beginning in 1982. ${ }^{14,15}$ The data include information such as an individual's age, gender, MSA of residence, race, vital status as of 2001, main transmission category, gender and sexual orientation. There are a total of 859,000 diagnoses contained in APIDS from January 1982 through December 2002. The key variables are defined in Table A.4.

The CDC put forth a great effort to ensure the quality and trustworthiness of these data. The case report forms that make up APIDS were filled out by health care providers or AIDS surveillance staff in health departments. Patients were interviewed to obtain information on their sexual orientation and behavioral patterns that could have been relevant to their diagnoses. The CDC would then follow up with many of these cases in order to remove duplicates, fix incomplete or incorrect reports, and update information as the patients' illness progressed. There are multiple studies attesting to the completeness of the APIDS data (Rosenblum et al., 1992; Lee et al., 2001).

There are a number of important features of the APIDS data which impact our analysis. First, there is substantial variation in the differences between the date of diagnosis and date the CDC obtains the case report. While the modal delay is only 1-2 months, the average is over 9 months. Report delay, and its implications, is discussed further in Appendix section A.7.2.

Next, if a patient had multiple possible transmission categories, they would be only counted in the category that appears first on the hierarchy. Therefore, crucially, heterosexual men who were both injection drug users and engaging in risky heterosexual sex would be listed as just being a drug user. This is an important point, as we use both transmission categories "IV Drug Use" and "Heterosexual Sex" for our identification of heterosexual men. In addition, while injection drug use was one of the first activities to be linked with the spread of HIV/AIDS, injection drug users still had substandard knowledge of the disease (CDC, 1992), particularly compared to homosexual men (Hingson et al., 1989).

The field that describes the surveillance definition of AIDS for each case works in a similar fashion. The pre-1985 definition is the original (and most severe) surveillance definition of AIDS. However, the CDC developed new, more inclusive, surveillance definitions of AIDS in 1985, 1987 and 1993 such that all cases that qualify for an earlier definition also qualify for a later definition. The way this variable is coded allows for easy comparisons between diagnoses, most importantly the severity.

Finally, the vital status of each patient is also recorded in APIDS. The CDC has the exact date of death

\footnotetext{
${ }^{14}$ It appears that the CDC uses the 1999 PMSA definitions and the 1990 NECMA definitions. While MSAs change over time, we make sure to capture the same counties over our sample period instead of MSAs for this reason.

$1517 \%$ of the APIDS cases do not have an associated MSA, but rather a general region. This represents those "in an MSA with less than 500,000 population, in a non-metropolitan area, or whose metropolitan area of residence is unknown, and for all pediatric patients." These are also dropped from the analysis.
} 
and survival time for each patient, but these data are confidential. Therefore, vital status is reported as a binary variable equal to 1 if the CDC had received a notice of death for the patient before the end of 1999. In other words, all cases diagnosed in January 2000 and onward are marked as alive for confidentiality purposes. However, if the CDC never obtained further information about a case, that patient would be denoted as alive in APIDS. These details are all elaborated on in more detail below.

\subsection{NCHS Multiple Cause of Death Data}

The National Center for Health Statistics (NCHS) maintains records of every death occurring in the United States. Using these records, researchers can identify when and where a person died as well as the causes of that person's death. We extract all records of HIV/AIDS related deaths from 1987 through 1995, and then aggregate a sum for each month and MSA combination in order to preform our analysis.

In addition to deaths related to HIV/AIDS, we also extract data on deaths related to overdose deaths in order to control for the prevalence of drug use in an MSA. ${ }^{16}$ Figure 2 shows the number of AIDS diagnoses and deaths per month in all the MSAs in the United States from 1982 to $2002 .{ }^{17}$ The upper red trend line represents the number of diagnoses while its lower, blue counterpart shows number of deaths as a result of AIDS. The vertical lines in Figure 2 represent the date used to beginning and end of the sample period, Johnson's announcement, and 24 months after the sample's end. Visually, it seems that a 24 month lag of AIDS deaths is highly predictive of its diagnoses. Further, a lag of 24 months gives us the highest $R^{2}$ (as opposed to any other number of lags) when trying to predict diagnoses using deaths. While imperfect, this visual evidence coupled with the $R^{2}$ is our justification for using AIDS related deaths in $t+24$ as a control for underlying AIDS prevalence in month $t .^{18}$

\subsection{Homosexual Population Index}

Walther and Poston Jr (2004) created indices for gay and lesbian partnering in large metropolitan areas in the United States by using data from the 1990 census. We use this measure to roughly estimate the population of homosexual men within an MSA since the data overlaps with our sample period and the indices cover a large majority of the MSAs the in our sample. ${ }^{19}$ In particular, we use their index which estimates the number of homosexual males per 100,000 males aged 18 and older. We couple this with yearly population data from the US Census Bureau's county estimates and adjust for MSA definition changes.

\subsection{Ryan White CARE Act}

Following the death of the American teenager and AIDS-education activist Ryan White, Congress passed the Ryan White CARE Act in August 1990. The goal of the program was to provide resources for medical

\footnotetext{
${ }^{16}$ In addition, we also collect Fryer Jr et al. (2013)'s Crack Index. Neither of these variables have any influence on our results and are therefore not discussed further.

${ }^{17}$ Each entry in APIDS contains a weighting adjustment because of delays in reporting. The first weight that is not equal to one appears in December 1997. We suspect that the severe drop off at the end of the time series is due to reporting delay.

${ }^{18}$ Lemp et al. (1990) report that the median survival for patients diagnosed with AIDS was 12.5 months, with a 5-year survival rate of 3.4\%. While insightful, these statistics were gathered between July 1981 and December 1987 - before the introduction of AZT treatment which was shown to prolong the lifespan of patients with AIDS (Fischl et al., 1987). In addition, Lee et al. (2001) reports that at least $60 \%$ of all cases diagnosed before 1993 had died after 24 months.

${ }^{19}$ We do not have index estimates for Ann Arbor MI, Colorado Springs CO, Columbia SC, Daytona Beach FL, Fort Wayne IN, Las Vegas NV, McAllen TX, Mobile AL, Orange County CA, San Juan PR, Sarasota FL, Stockton CA, Vallejo CA, Ventura CA, Witchita KS, Wilmington DE, or Youngstown OH. This accounts for $19 \%$ of the sample.
} 
Figure 2: AIDS Diagnoses \& Deaths in the CDC's selected MSAs

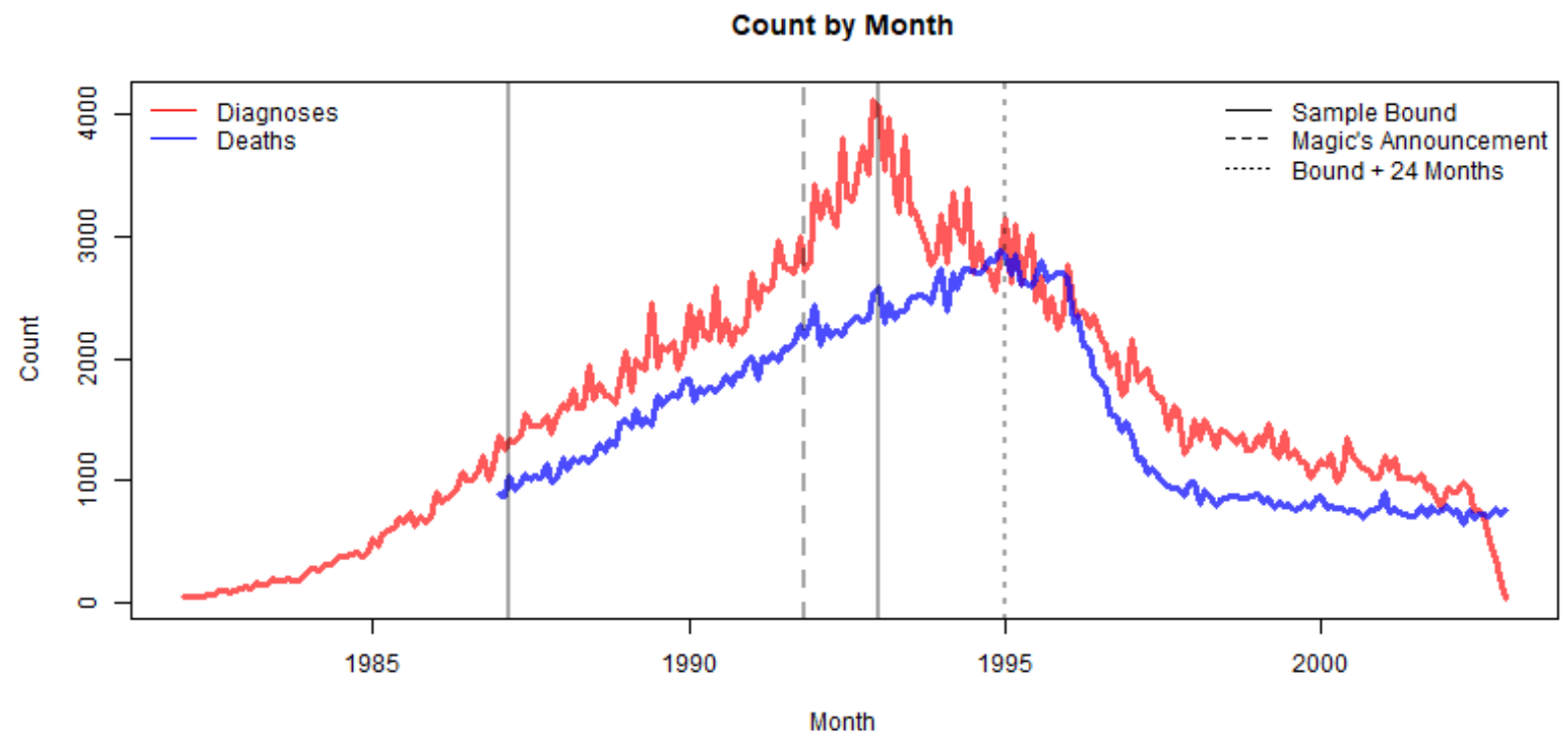

Note: Figure represents the number of AIDS diagnoses and deaths by month. The solid vertical lines represent the beginning and end of the study sample, while the dashed lines represent Johnson's diagnosis and the approval of HAART from left to right.

treatment in areas of the country where HIV and AIDS were most prevalent. The first grants were distributed midway through 1991, and by the end of the year the CARE act distributed $\$ 220$ million to both researchers and cities which struggled to contain the virus. ${ }^{20}$

Over the course of our sample, the funding from the Act was dispersed to 25 MSAs with the highest prevalence of HIV, and was the largest federally funded program in the United States for PWAs. Table 2 breaks down the number of MSAs with NBA teams and CARE funding. Perhaps surprisingly, this is a relatively balanced set of MSAs. We discuss the filtering of these MSAs below.

Table 2: CARE Funding and NBA Team breakdown

\begin{tabular}{ccc}
\hline & NBA Team & No NBA Team \\
\hline CARE Funding & 11 & 9 \\
No CARE Funding & 13 & 26 \\
\hline
\end{tabular}

Note: Table contains counts of MSAs used in the main analysis sample by whether they had an NBA team and/or CARE funding.

\subsection{Time Frame}

We begin our sample in September 1987 in order to account for two important milestones in the timeline of the AIDS pandemic. First, in March 1987, the FDA approved the first medication for patients with

\footnotetext{
${ }^{20}$ Dillender (2021) demonstrates that the CARE Act successfully reduced AIDS related deaths in those cities that secured funding.
} 
HIV/AIDS: azidothymidine (AZT). Early treatment with AZT was shown to delay the progression of AIDS via an increase in patient's white blood cell counts. ${ }^{21}$ Second, there is a change in surveillance definitions that takes place in August 1987. Beginning the sample in September, we avoid any oddities that come with the introduction of a new definition and large change in medical treatment.

We then end our sample after December 1992, near the peak of AIDS diagnoses in the United States. We first do so to avoid any large medical advancements which substantially change life expediencies of living with AIDS in order to maintain a homogeneous time-to-death period over the time window. At the end of 1995, the FDA approved the first protease inhibitor which lead to a new era of highly active antiretroviral therapy (HAART). This drug dramatically improved the treatment and lifespan expectations for PWAs. As discussed earlier, we utilize AIDS related deaths two years into the future in order to proxy for the underlying population with HIV/AIDS which makes the end of 1992 a desirable cutoff. Second, the definition of what constitutes an AIDS diagnosis again changed starting in January 1993 as the medical community learned more about the disease. These five-and-a-half years (September 1987 through December 1992) represent a time period in which options for treatment and testing remained relatively constant over time.

Figure 3: Timeline of Important AIDS Related Events

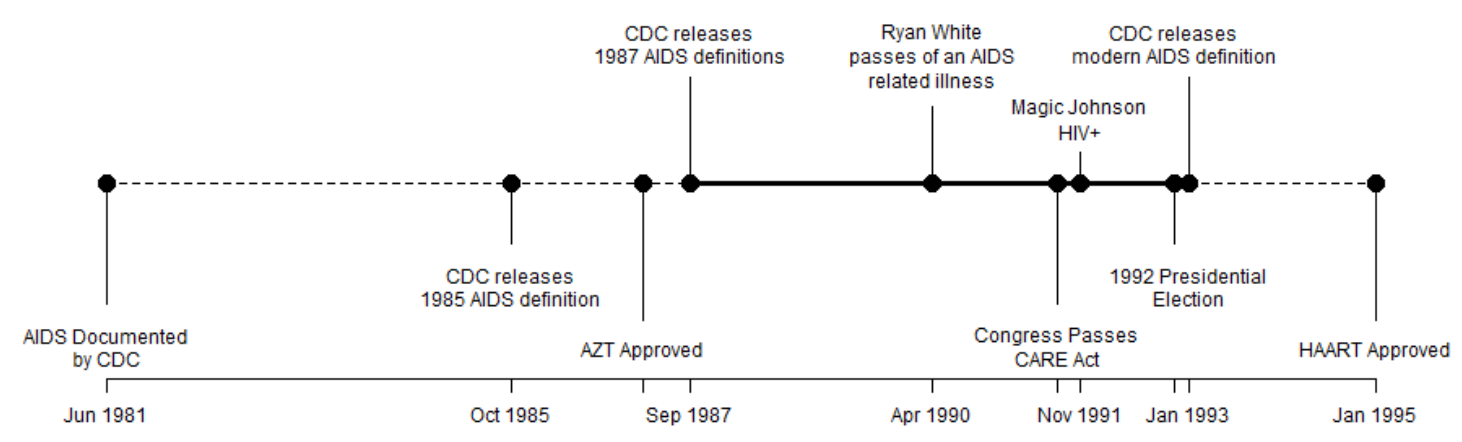

Note: Figure represents major events in the AIDS landscape relevant to this study. These events include the first documentation of AIDS, key changes in AIDS surveillance definitions, medical advancements and cultural events. The solid horizontal line represents the time period studied.

\subsection{Analysis Samples}

We consider a select group of citieis for the analysis. We begin with 104 MSAs, and order these by the number of overall diagnoses. There are a number of significant outliers which we drop in order to maintain a reasonable distribution. The significant outliers on the right-hand side of the distribution are New York (28,097 cases), Los Angeles (13,077 cases) and San Francisco (11,270 cases). We also exclude the 40 MSAs which were least impacted by the AIDS pandemic. Notably, none of these later MSAs include an NBA Franchise. Finally, we remove San Juan and Honolulu in order to only analyze MSAs in the contiguous United States. ${ }^{22}$ We are left with 59 MSAs in our main analyses.

\footnotetext{
${ }^{21}$ However, despite the early excitement, the side effects of the drug that was originally developed as a form of chemotherapy proved to be unbearable for many. For example, Hamilton et al. (1992) demonstrated that the side effects associated with the usage of AZT could be severe enough to offset its impact on survival.

${ }^{22}$ Honolulu is dropped with the bottom 40.
} 
There are seven total surveillance definitions in APIDS: the pre-1985 definition (pre-1985), the 1985 definition, the 1987 Definitive (1987-D) definition, 1987 Presumptive (1987-P) definition, the 1993 Definitive definition, 1993 Presumptive definition and the 1993 severe immunosuppressive definition. We remove all cases diagnosed under the 1993 definitions. These diagnoses would be retroactive since we end the sample in December 1992. We also remove diagnoses meeting the 1985 definition as this one category makes up just over $3 \%$ of the remaining four surveillance definitions. Each successive surveillance definition is completely inclusive of all previous, and definitions from earlier years are usually more advanced progressions of AIDS than definitions from later years. ${ }^{23}$

The first of the three surveillance definitions left is the pre-1985 definition. Cases meeting the criteria for this definition are the most severe and progressed of any later definition. The remaining two, the 1987 definitive and presumptive definitions, have relatively similar mortality rates although differ in how they are diagnosed. The definitive definition relies specifically on blood work, which lends itself to our hypotheses regarding asymptomatic diagnoses. The presumptive definition is, by construction, designed for symptomatic individuals with obvious signs of AIDS.

In Table 3, we break down the number of non-adolescent, non-pediatric cases (ages 20+) from September 1987 through December 1992 in our main sample of MSAs by reported sexuality and transmission category. ${ }^{24}$ The diagnoses with transmission categories relevent to our identification strategy and hypotheses are denoted with $\mathrm{a}^{+}$. We hypothesize that Johnson likely had the greatest impact on heterosexual men, particularly those in NBA MSAs. Therefore, one of our treated groups is comprised of heterosexual men with transmission categories of Heterosexual Sex and IV Drug Use. The most comparable group to these men are homosexual men with transmission categories "Homosexual Sex" and "Homosexual Sex \& IV Drug Use".

Table 3: Tabulation of Sexuality and Relevant Transmission Categories, Full Sample

\begin{tabular}{l|cccc}
\hline & \multicolumn{4}{|c}{ Sexual Identity and Gender } \\
Transmission Category & Homosexual & Bisexual & Heterosexual & Female (all) \\
\hline Homosexual Sex & $55,448^{+}$ & 12,798 & 58 & 0 \\
IV Drug Use & 1 & 0 & $18,322^{+}$ & 7,452 \\
Homosexual Sex \& IV Drug Use & $5,755^{+}$ & 3,183 & 145 & 0 \\
Hemophilia & 0 & 0 & 692 & 23 \\
Heterosexual Sex & 0 & 0 & $2,899^{+}$ & 5,122 \\
Blood Transfusion & 0 & 0 & 1,056 & 772 \\
Unidentified / Not Reported & 0 & 0 & 3,186 & 939 \\
\hline Total & 61,203 & 0 & 21,221 & 0 \\
\hline
\end{tabular}

Note: Table breaks down the APIDS cases in the temporal and spatial units of analysis. Individuals included used for the primary analyses are marked with ${ }^{+}$.

\subsubsection{Diagnoses}

To test the hypothesis that Johnson's announcement increased diagnoses, we count the number of diagnoses for each MSA-by-Sexuality group (hereinafter: community) by month. We augment these counts with yearly population numbers adjusted by Walther and Poston Jr (2004)'s homosexual population index, monthly

\footnotetext{
${ }^{23}$ For example, $90 \%$ of diagnoses qualifying for the Pre-1985 surveillance definition were reported to have died compared to $86.5 \%$ for both of the 1987-P and 1987-D surveillance definitions.

${ }^{24}$ We only keep diagnoses of patients labeled as non-Hispanic White, non-Hispanic Black and Hispanic. These three race categories make up over $99 \%$ of diagnoses.
} 
future AIDS deaths, if the MSA contains an NBA team and if it obtained funding through CARE.

We present summary statistics of this constructed sample in Table 4. The average number of diagnoses in a community is 11 . Therefore, the average number of diagnoses for entire MSAs is about 22, since we observe MSAs twice per month (one observation per community). This number corresponds to the average AIDS Deaths in $t+24$ of 23.1. In addition, about one fifth of observations come after Magic Johnson's announcement, $40 \%$ are for communities with an NBA team and $33 \%$ obtained CARE funding.

Table 4: Summary Statistics for Diagnoses Sample

\begin{tabular}{lcccc}
\hline & Mean & St. Dev. & Min & Max \\
\hline Heterosexual & 0.500 & 0.500 & 0 & 1 \\
Diagnoses & 10.999 & 13.883 & 0 & 104 \\
Post Magic & 0.219 & 0.413 & 0 & 1 \\
NBA & 0.407 & 0.491 & 0 & 1 \\
CARE & 0.339 & 0.473 & 0 & 1 \\
Homosexual Population Index & 117.407 & 101.546 & 37.400 & 773 \\
AIDS Deaths & 23.109 & 20.522 & 0 & 128 \\
\hline
\end{tabular}

Note: Table contains summary statistics for the sample used to estimate Magic Johnson's impact on diagnoses. There are 7,552 community-by-month observations. Of note, we report the average diagnoses across communities, which is about 11. However, of course there exists substantial heterogeneity in diagnoses across communities. For example, heterosexual communities had significantly less diagnoses on average (5.66) compared to homosexual (16.34) communities.

\subsubsection{Composition of Diagnoses \& Mortality}

We use the disaggregated data in Table 3 to address the hypotheses surrounding mortality. We present summary statistics for this sample in Table 5 . Of the 82,424 diagnoses, about three quarters (72.6\%) qualify for the Pre-1985 surveillance definition. The CDC had received a notification of death for $89 \%$ of all diagnoses. $28 \%$ of diagnoses occurred following Johnson's announcement. $57 \%$ occurred in NBA MSAs while only $40 \%$ of MSAs are home to an NBA team.

Table 5: Summary Statistics for Mortality Sample

\begin{tabular}{lcccc}
\hline & Mean & St. Dev. & Min & Max \\
\hline Alive & 0.11 & 0.313 & 0 & 1 \\
Post Magic & 0.28 & 0.449 & 0 & 1 \\
Heterosexual & 0.257 & 0.437 & 0 & 1 \\
Report Delay & 9.422 & 14.994 & 0 & 180 \\
NBA & 0.574 & 0.494 & 0 & 1 \\
CARE & 0.506 & 0.500 & 0 & 1 \\
Pre-1985 Defintion & 0.726 & 0.446 & 0 & 1 \\
1987 Presumptive Def. & 0.126 & 0.332 & 0 & 1 \\
1987 Definitive Def. & 0.148 & 0.355 & 0 & 1 \\
White & 0.59 & 0.492 & 0 & 1 \\
\hline
\end{tabular}

Note: Table contains summary statistics for the sample used to estimate Magic Johnson's impact on mortality. There are 82,424 case observations used. 


\section{Empirical Strategy}

This section discusses the empirical strategies used to test the hypotheses put forth in previous sections. We test for changes in the number of diagnoses, the composition of diagnoses and the outcomes of these diagnoses.

\subsection{Diagnoses}

We observe each community in our sample by month. We transform the counts of diagnoses in order to estimate changes in the rates of diagnoses. While this is traditionally done in the literature by using logarithms, we instead make use of the inverse hyperbolic sine. This transformation allows for the same interpretation as logarithms while empirically accommodating for zeros. We include prevalence and population estimates as control variables rather than part of the dependent variable due to the fact that our population estimates are drastically different in magnitude while diagnoses are much closer in scale. In Equation (1), we specify a reduced form model for the rate of AIDS diagnoses $D$ in community $c$ in time period $t$ as follows:

$$
\begin{aligned}
\sinh ^{-1}\left(D_{c t}\right)=\alpha_{c}+\beta_{c} \text { Trend }+\delta_{0}\left(\text { Magic }_{t}\right)+ & \delta_{1}\left(\text { Heterosexual }_{c} \mathrm{x} \mathrm{Magic}_{t}\right)+\delta_{2}\left(\mathrm{NBA}_{c} \mathrm{x} \text { Magic }_{t}\right) \\
& +\delta_{3}\left(\mathrm{NBA}_{c} \mathrm{x} \text { Heterosexual }_{c} \mathrm{x}_{\mathrm{Magic}_{t}}\right)+\phi X_{c t}+\epsilon
\end{aligned}
$$

where the time trend is measured in number of months since Magic's announcement. For interpretability, November 1991 is set as $0 . \alpha_{c}$ and $\beta_{c}$ represents a fixed effect and trend for each community. The variable Magic represents a vector of 0 for observations before November 1991 and 1 including and after November 1991. To test the hypothesis that any impact due to Johnson's announcement could be different based on preannouncement knowledge of AIDS, we use an indicator variable, Heterosexual, equal to one if the observation is for a heterosexual community and equal to zero otherwise. To test the hypothesis that familiarity with Johnson could also be an important factor, we include an indicator variable, $N B A$, equal to 1 if there is an NBA team in the MSA and zero otherwise.

Since we do not know the number of people who followed the NBA, nor the actual level of AIDS knowledge, within each community, these parameters both represent intents to treat. We allow each of these variables to interact with one another in a triple difference-in-differences framework. We neither write down, nor estimate, the interaction between $N B A$ and Heterosexual, however, because this interaction is absorbed by the aforementioned community fixed effects.

$X_{c t}$ is a vector of control variables such as population, AIDS related deaths two years into the future, overdose deaths two years into the future, month fixed effects, and CARE funding. We control for CARE funding by including an indicator variable for MSAs that received funding from the CARE Act in 1991, 1992 or 1993. Since the initial funding occurred right around Johnson's announcement, we interact this indicator with Magic, Heterosexual, and Magic $\times$ Heterosexual. This can be thought of similarly to the NBA variable, but ultimately out of the scope of this study. ${ }^{25}$

For interpretation, our parameters of interest are each $\delta_{j} . \delta_{0}$ represents the effect on all groups following Johnson's announcement. A positive estimate would suggest an increase in the rate of diagnoses for homo-

\footnotetext{
${ }^{25}$ Dillender (2021) presents evidence that CARE funding was helpful in reducing deaths due to AIDS, but does so in years outside of our sample period.
} 
sexual communities in non-NBA MSAs. $\delta_{1}$ represents the parameter associated with our first hypothesis that Johnson's impact would have been greater for a group, heterosexual men, unfamiliar with their risk relative to those, homosexual men, who were indeed familiar. A positive coefficient would suggest an additional increase in the rate of AIDS diagnoses on top of the estimate of $\delta_{0} . \delta_{2}$ can be interpreted similarly to $\delta_{1}$, although this tests whether Johnson's impact was different among communities in NBA MSAs. Finally, $\delta_{3}$ is a triple difference coefficient. This measures the differential impact of heterosexual communities in NBA MSAs vs non-NBA MSAs or heterosexual communities in NBA MSAs vs homosexual communities in NBA MSAs.

\subsection{Composition of Diagnoses}

We examine both the composition and outcomes of the increased diagnoses in order to rule out factors which might have driven the effect from equation 1 rather than Johnson's announcement. First, we model the probability of a diagnosis qualifying for each specific surveillance definition. Given the three diagnosis categories (Pre-1985, 1987-Presumptive, and 1987-Definitive) we consider, there should be an increase in the probability of the 1987-Definitive diagnosis compared to the 1987-Presumptive one. A presumptive diagnosis indicates that a patient has obvious symptoms of AIDS such as wasting disease, Kaposi sarcoma, etc. However, for those men without symptoms, only a blood test (or definitive diagnosis) would be able to identify their positive AIDS status. Therefore, if there were to be "early" diagnoses, they would fall into the 1987-Definitive surveillance definition. We model the probability of person $i$ in community $c$ in time period $t$ being diagnosed into surveillance definition ("SD") $j$ using the following equation:

$$
\begin{aligned}
P\left(S D_{j}\right)_{i c t}=\alpha_{c}+\delta_{0}\left(\text { Magic }_{t}\right) & +\delta_{1}\left(\text { Heterosexual }_{i} \times \operatorname{Magic}_{t}\right)+\delta_{2}\left(\mathrm{NBA}_{i} \mathrm{x} \text { Magic }_{t}\right) \\
& +\delta_{3}\left(\mathrm{NBA}_{i} \times \text { Heterosexual }_{i} \times \operatorname{Magic}_{t}\right)+\phi X_{i c t}+\epsilon_{i c t}
\end{aligned}
$$

where each variable is defined the same way as in Equation (1) except for $X_{i c t}$. This matrix contains control indicators for age, race, transmission categories, and fixed effects for diagnosis month and report month. As a note, because we estimate one regression of each $S D_{j}$ in this section, the corresponding coefficients across these regressions will sum to 1 . This is due to the probability of being in one of these categories must equal 1. In other words, a substitution towards any one category must imply a substitution away from at least one other category.

\subsection{Mortality}

Diagnosing AIDS early on in the course of the disease is one of the biggest factors in extending lifespan. Therefore, if Johnson's announcement encouraged men to get tested earlier than they would have otherwise, we would expect an increase in the average lifespan following a diagnosis for those men in communities with the largest increase in diagnoses. Further, we would expect to see this increase in the group being diagnosed via blood tests instead of presumptively. Unfortunately, the public version of APIDS does not have the ability to measure time to death following a diagnosis directly. Rather, the variable that is contained tells whether or not the CDC had received a notice of the patients death. Therefore, we model the probability of this event with the following equation: 


$$
\begin{aligned}
P\left(\text { Alive }_{i c t}=\alpha_{c}+\delta_{0}\left(\text { Magic }_{t}\right)\right. & +\delta_{1}\left(\text { Heterosexual }_{i} \mathrm{x} \mathrm{Magic}_{t}\right)+\delta_{2}\left(\mathrm{NBA}_{i} \mathrm{x} \text { Magic }_{t}\right) \\
& +\delta_{3}\left(\mathrm{NBA}_{i} \mathrm{x} \mathrm{Heterosexual}_{i} \mathrm{x}^{\text {Magic }_{t}}\right)+\phi X_{i c t}+\epsilon_{i c t}
\end{aligned}
$$

Similar to the effect for diagnoses, these effects should be largest immediately following the shock and taper off over time. We also estimate the triple difference coefficient for each quarter following Johnson's announcement to test for these dynamics.

\section{$7 \quad$ Results}

\subsection{Diagnoses}

We begin our main analysis by presenting an unconditional time series of the average inverse hyperbolic sine of diagnoses for the four groups of communities. In Figure 4, we observe an immediate but temporary deviation in the time series of heterosexual men in NBA MSAs from heterosexual men in non-NBA MSAs. However, after about 9 or 10 months, the time series appears to return to it's previous relative location. There is very little change if any for homosexual men.

Table 6: AIDS Diagnoses Following Magic Johnson

\begin{tabular}{lcccc}
\hline & \multicolumn{4}{c}{ sinh $^{-1}$ (Diagnoses) } \\
\hline Magic & -0.040 & -0.026 & -0.051 & -0.042 \\
& $(0.030)$ & $(0.028)$ & $(0.047)$ & $(0.049)$ \\
Magic x Heterosexual & $0.102^{* *}$ & & 0.077 & 0.081 \\
& $(0.043)$ & & $(0.063)$ & $(0.066)$ \\
Magic x NBA & & $0.091^{* *}$ & 0.003 & -0.010 \\
& & $(0.045)$ & $(0.059)$ & $(0.061)$ \\
Magic x Heterosexual x NBA & & & $0.198^{* *}$ & $0.199^{* *}$ \\
& & & $(0.082)$ & $(0.084)$ \\
\hline Num.Obs. & 7,552 & 7,552 & 7,552 & 7,296 \\
MSA-by-Sexuality Trend & Yes & Yes & Yes & Yes \\
CARE & No & No & Yes & Yes \\
Population & No & No & No & Yes \\
AIDS Deaths \\
R2 & No & No & No & Yes \\
R2 & 0.853 & 0.853 & 0.853 & 0.855 \\
\hline \multirow{2}{*}{$<0.1, * *$} \\
\hline
\end{tabular}

$* \mathrm{p}<0.1,{ }^{* *} \mathrm{p}<0.05, * * * \mathrm{p}<0.01$

Note: These OLS regressions estimate the effect of Magic Johnson's announcement on the rate of AIDS diagnoses in select MSAs. Each regression contains fixed effects for MSA-by-Sexuality and month of diagnosis. In addition, specific MSA-by-Sexuality trends are also controlled for. Standard errors are clustered at the MSA-by-Sexuality level. Results are qualitatively similar when raw diagnosis counts are used (OLS \& Poisson) instead of the inverse hyperbolic sine transformation. 
Figure 4: Time Series of Group Definitions

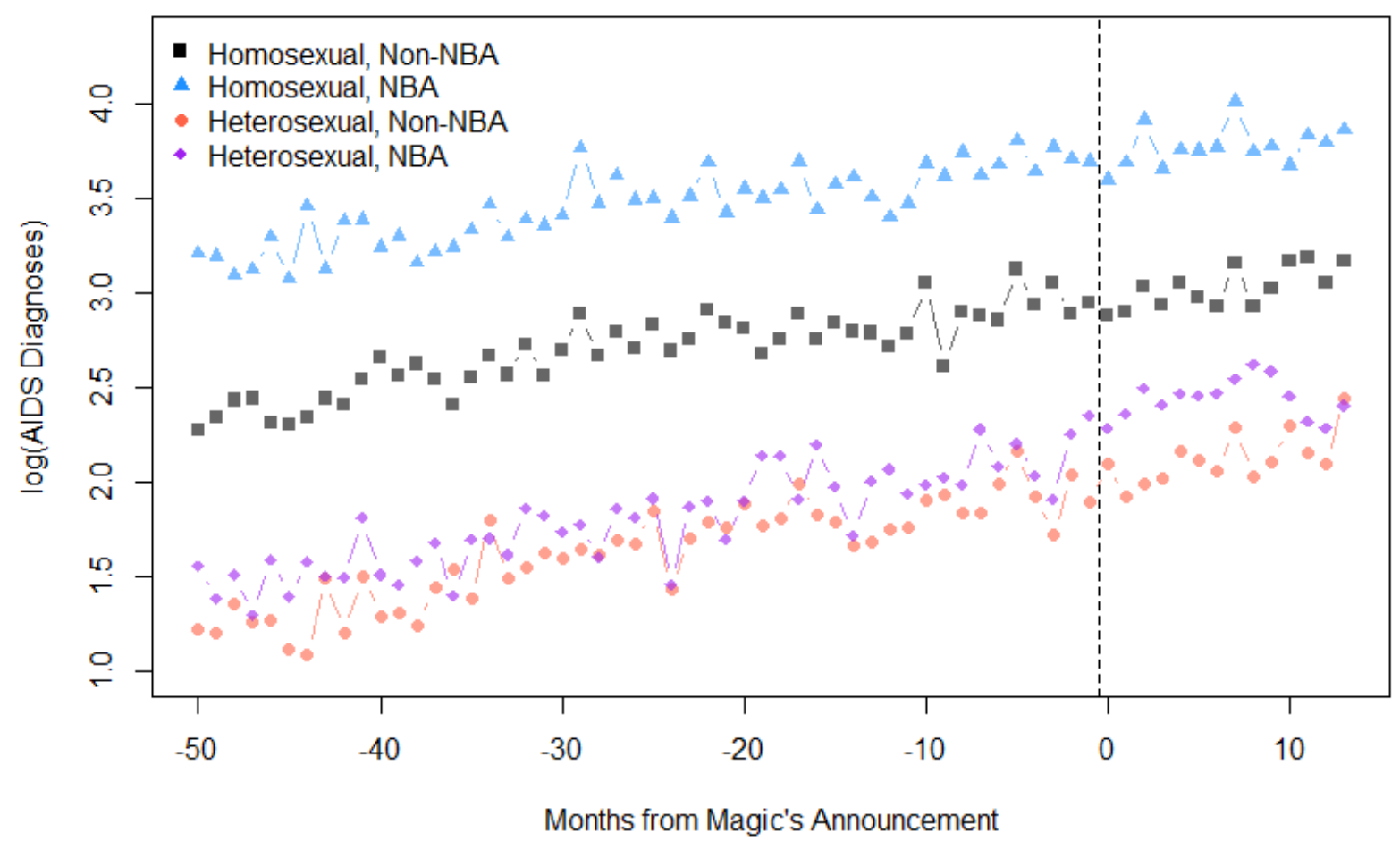

Note: Figure represents time series of the four groups studied: homosexual and heterosexual men in NBA and non-NBA MSAs. The $\mathrm{y}$-axis represents the average of the inverse hyperbolic sine of diagnoses. The $\mathrm{x}$-axis is denoted in months from Magic Johnson's announcement. 
Next, we estimate the parameters in Equation (1) and present the results in Table 6. The first two columns present results from models slightly less complex than the triple difference model in Equation 1. Instead, the first column ignores information about pre-announcement exposure to Johnson $(N B A)$ and the second column ignores information about the sexuality of the community (Heterosexual). The parameter estimates in these first two columns suggest an approximate $10 \%$ increase in the rate of AIDS diagnoses for heterosexual communities and communities in MSAs with an NBA team, respectively. These results support both hypotheses that communities with less knowledge and higher exposure would react the most. Interestingly, the magnitudes of the coefficient estimates are nearly identical, suggesting similar effects. In addition, the parameter estimate for Magic is insignificant, suggesting no effect for homosexual / non-NBA communities, depending on the column.

Columns 3 and 4 estimate the triple difference model outlined previously. Column 4 only differs in its controls. The coefficient estimate for Magic remains insignificant. However, both coefficient estimates for Magic $\times N B A$ and Magic $\times$ Heterosexual are insignificant. Still, Magic $\times$ Heterosexual is still consistently positive whereas Magic $\times N B A$ is almost exactly zero. The effect is almost completely absorbed into the triple difference parameter. Therefore, the majority of the impact of Johnson's announcement on the rate of diagnoses was in heterosexual communities in MSAs with NBA teams. The magnitude of the estimates are about .2, which suggests a $20 \%$ increase in the rate of diagnoses for these communities. This equates to about 1 extra diagnosis per community per month on average.

Finally, in Figure 5, we reestimate the triple difference parameter in column (4) in Table 6 for each quarter following Johnson's announcement. We recode the months such that the first quarter is made of November, December and January, and so on. This allows us to see how the coefficient estimate evolves over time. We observe a large, positive effect for the first three quarters, but this trails off by the end of the sample. This is suggestive of a temporal shift in diagnoses. Again, since the number of PWAs is relatively fixed, an increase in time $t$ should correspond with a decrease in time $t+j$, or at least a return to trend.

\subsection{Composition of Diagnoses}

We begin this portion of the analysis by analyzing changes in the probability of being diagnosed into a certain AIDS surveillance definition relative to all others. Table 7 displays the estimated coefficients from Equation

2. The parameter estimates in each row should sum to zero since a substitution away from one surveillance definition category implies a substitution towards another. The results from the first three columns suggest a substitution away from presumptive diagnoses and (mostly) towards the definitive diagnoses. While the triple-difference coefficient (Magic $\times N B A \times$ Heterosexual) in column 3 is insignificant, it is $75 \%$ of the triple difference coefficient in column 2. These results provide some evidence of a change in the composition of the diagnoses for heterosexual men in NBA MSAs being after Johnson's announcement. 
Figure 5: Impact of Magic Johnson on Diagnoses Over Time

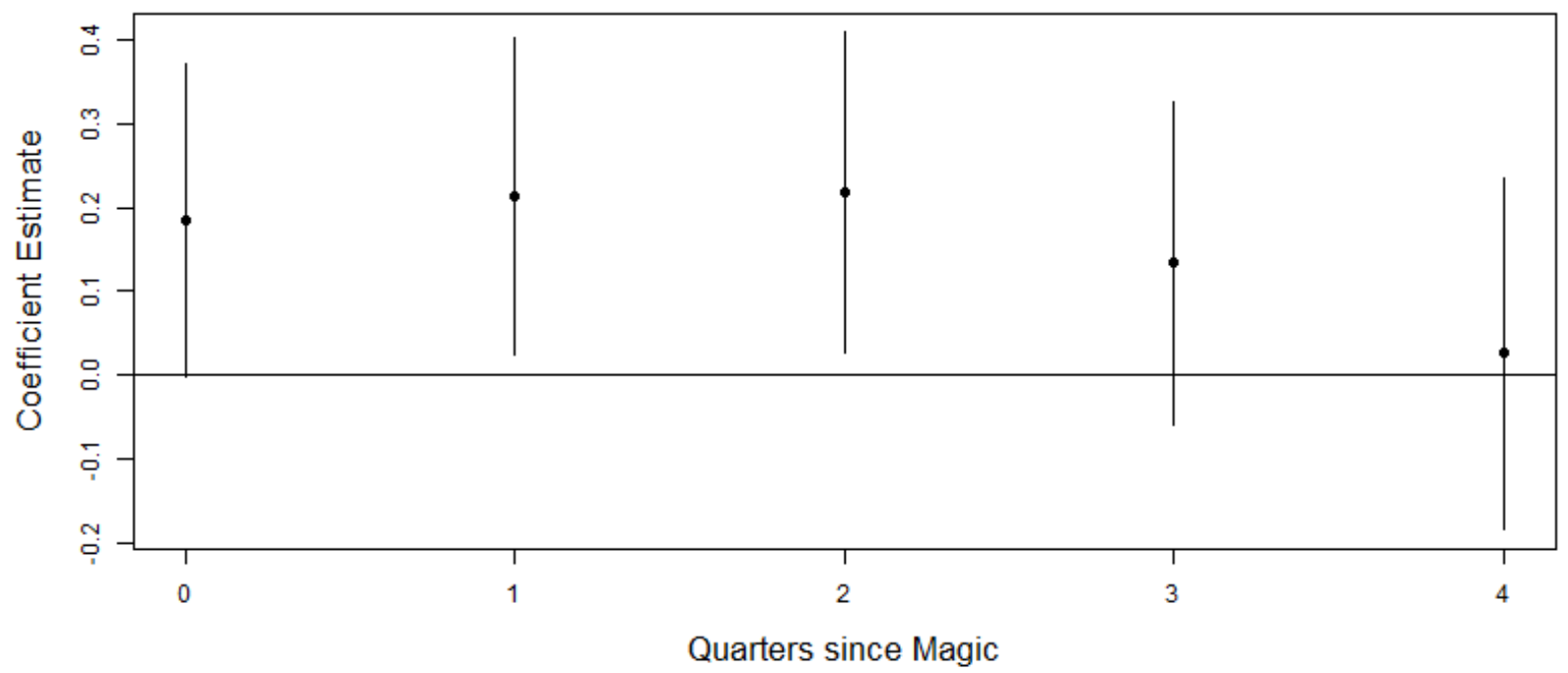

Note: Figure represents the evolution of Johnson's effect on heterosexual men in NBA MSAs (triple difference coefficient) over time. This uses the specification in Column (4) of Table 6.

Table 7: Probability of AIDS Surveillance Definitions Following Magic Johnson

\begin{tabular}{lccc}
\hline & $\mathrm{P}($ Pre-1985) & $\mathrm{P}(1987-\mathrm{P})$ & $\mathrm{P}(1987-\mathrm{D})$ \\
\hline Magic x Heterosexual & -0.002 & 0.009 & -0.008 \\
& $(0.020)$ & $(0.015)$ & $(0.013)$ \\
Magic x NBA & 0.013 & -0.001 & -0.012 \\
& $(0.015)$ & $(0.012)$ & $(0.010)$ \\
Magic x NBA x Heterosexual & 0.011 & $-0.040^{* *}$ & 0.029 \\
& $(0.025)$ & $(0.017)$ & $(0.021)$ \\
\hline Num.Obs. & 82,424 & 82,424 & 82,424 \\
R2 & 0.104 & 0.079 & 0.047 \\
\hline
\end{tabular}

$* \mathrm{p}<0.1,{ }^{* *} \mathrm{p}<0.05,{ }^{* * *} \mathrm{p}<0.01$

Note: These LPM regressions estimate the impact of Magic Johnson's announcement on types of diagnoses. Each regression contains fixed effects for MSA-by-Sexuality, race, age, month of diagnosis, month of report, and transmission category. Standard errors are clustered at the MSA-by-Sexuality level.

Following the final step in the previous subsection, we reestimate the triple difference coefficient for each quarter following Johnson's announcement. Figure 6 reveals a relatively static, zero effect for the pre-1985 surveillance definition category. However, there is a clear pattern that emerges in the two 1987 definitions. The middle panel of the figure shows that there was an immediate decrease in the probability of being diagnosed via the presumptive definition. However, this decrease returns to zero after about 9 months. The 
9 month effect in this result corresponds to the 9 month result in the previous subsection. On the other hand, there is an opposite effect for being diagnosed using the 1987 definitive surveillance definition. There is an immediate increase in the probability following Johnson, but this also diminishes with time. These two panels support the hypothesis of a temporal shift. Again, definitive diagnoses are the result of a laboratory blood test whereas presumptive diagnoses are the result of visual symptoms, often when someone is too sick for a blood test or already symptomatic. Of these two categories, if one was going to be tested early, they would be diagnosed using the definitive diagnosis.

Figure 6: Changes in Composition of Surveillance Definitions Over Time
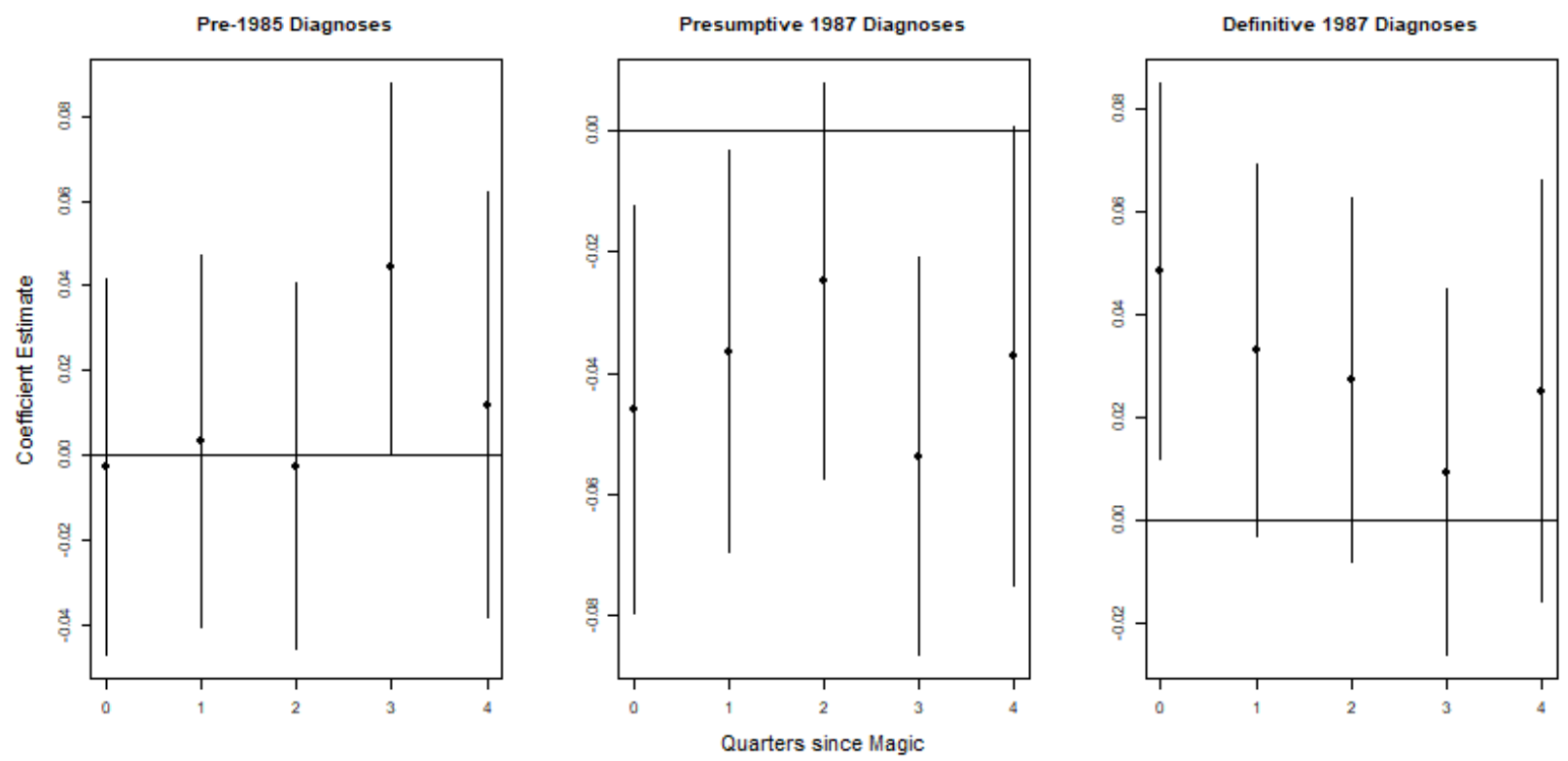

Note: Figure represents the evolution of Johnson's effect on the substitution towards/away from surveillance definitions over time for heterosexual men in MSAs with NBA teams. This uses the specifications in Table 7.

\subsection{Mortality}

If these diagnoses were occurring earlier than they would have otherwise, there should be differences in outcomes. Specifically, these early diagnoses should be able to live longer with AIDS, even without access to treatment, ceterus parabus. Unfortunately, as stated before, we do not have access to the variable detailing the month of death. Rather, we only have a variable that tells whether or not the patient had died. Using this as the outcome, we estimate Equation 3 and present the key parameter estimates in Table 8. Column (1) uses the entire sample, while Columns (2) through (4) uses a subset of the separate surveillance definitions. Column (1) finds a negative effect for heterosexual men in non-NBA MSAs. This, however, is offset by the positive effect for heterosexual men in NBA MSAs. While the overall effect is zero, it is still important that whatever is generating the negative effect in non-NBA MSAs is not effecting heterosexual men in NBA MSAs. Further, the positive effect for heterosexual men in NBA MSAs is being driven by those men being diagnosed with the definitive 1987 surveillance definition. This result supports the previous finding of an increase in the probability of being diagnosed with the definition definition. 
Table 8: AIDS Mortality Following Magic Johnson

\begin{tabular}{lcccc}
\hline & \multicolumn{4}{c}{ P(Alive in 2000) } \\
\hline Magic x Heterosexual & $-0.032^{* * *}$ & $-0.023^{* *}$ & $-0.049^{*}$ & $-0.049^{*}$ \\
& $(0.012)$ & $(0.010)$ & $(0.026)$ & $(0.026)$ \\
Magic x NBA & -0.005 & -0.005 & 0.021 & -0.020 \\
& $(0.009)$ & $(0.007)$ & $(0.023)$ & $(0.015)$ \\
Magic x NBA x Heterosexual & $0.034^{* *}$ & 0.018 & 0.027 & $0.073^{* * *}$ \\
& $(0.015)$ & $(0.013)$ & $(0.032)$ & $(0.026)$ \\
\hline Num.Obs. & 82,424 & 59,802 & 10,424 & 12,198 \\
Surveillance Definitions & All & Pre- 1985 & $1987-\mathrm{P}$ & $1987-\mathrm{D}$ \\
NA & NA & NA & NA & NA \\
R2 & 0.087 & 0.096 & 0.104 & 0.116 \\
\hline
\end{tabular}

${ }^{*} \mathrm{p}<0.1,{ }^{* *} \mathrm{p}<0.05,{ }^{* * *} \mathrm{p}<0.01$

Note: These regressions estimate the effect of Magic Johnson's announcement on mortality. Each regression contains fixed effects for MSA-by-Sexuality, race, age, month of diagnosis, month of report, and transmission category.Standard errors are clustered at the MSA-bySexuality level.

Once again, we estimate how these coefficients change over time. We observe a relatively flat, insignificant effect for the pre-1985 and presumptive 1987 surveillance definitions. However, there are three quarters of large effects for diagnoses meeting the definitive 1987 diagnosis. This is similar in that the previous results also had increases for the first three quarters after Johnson's diagnosis which zeroed out for the remaining two quarters. 
Figure 7: Changes in Mortality by Definition Over Time
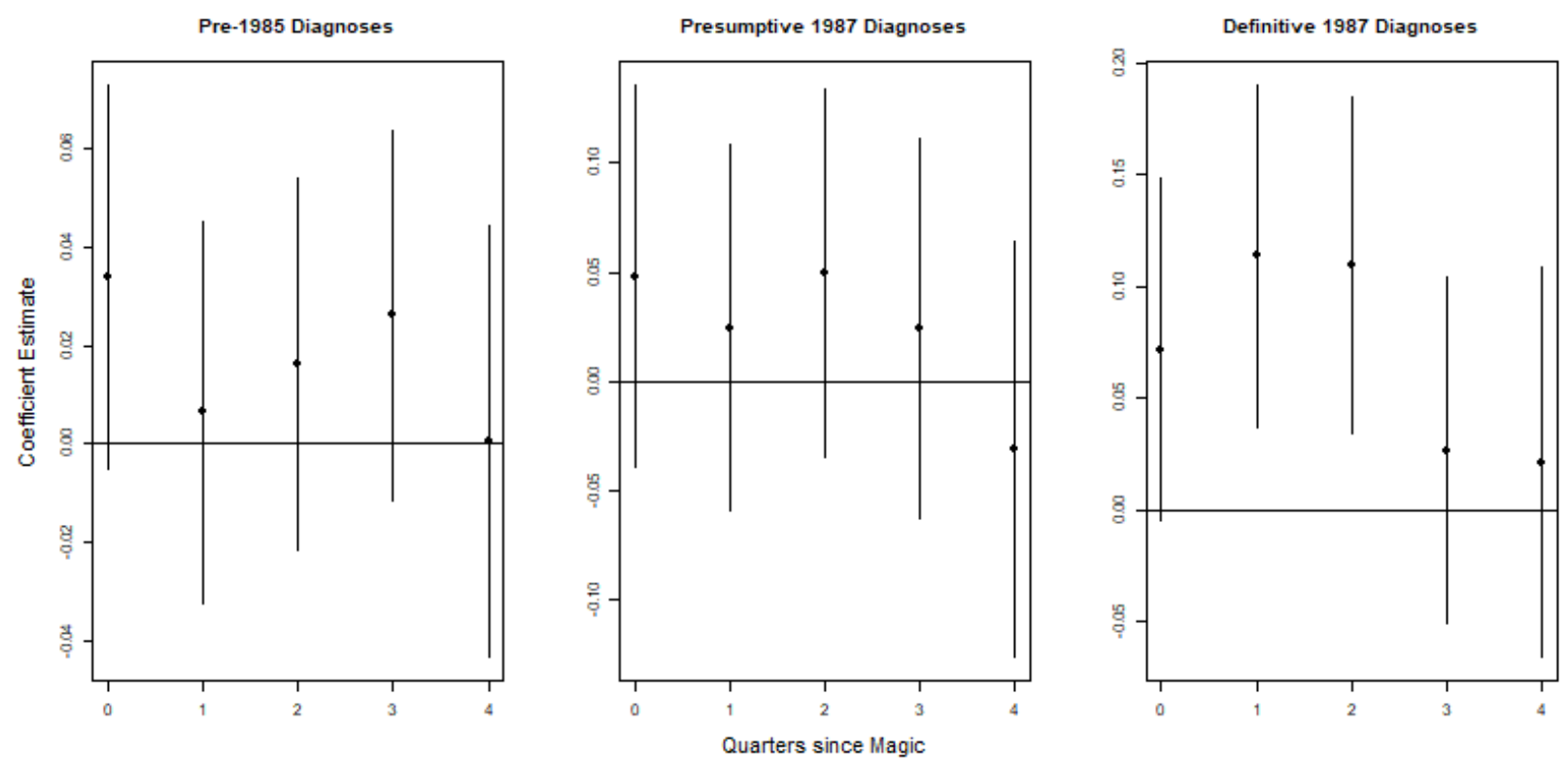

Note: Figure represents the evolution of Johnson's effect on the mortality of different surveillance definitions for heterosexual men in MSAs with NBA teams. This uses the specifications in Table 8.

\subsection{Heterogeneous Effects}

Next, we test for heterogeneous effects across our mortality sample. We test for differences between races, ages and transmission categories. We only present results graphically, however, since there is not much variation along these dimensions for changes in surveillance definition composition. We present the relative coefficient estimates and their respective 95\% confidence intervals in Figure 8.

We first decompose the triple difference coefficient in columns 1 and 4 in Table 8 by race. We observe a null result for diagnoses of White patients, but positive and significant results for both Black and Hispanic patients. This is true for both the full sample as well as the sub-sample of only 1987 definitive diagnoses. This is important heterogeneity since Magic Johnson is African American. Studies such as Kalichman and Hunter (1992) and Larson et al. (2005) find that celebrity disclosure of disease focuses the attention of those personally afflicted, but heterogeneous effects exist with regards to who seeks medical treatment, as those affected by the messaging tend to align demographically with the celebrity. Casey et al. (2003) finds further evidence regarding how minority populations felt about Johnson, citing (Flora et al., 1996) and their finding that people trusted Magic Johnson over a wider range of other black celebrities.

Next, we examine the mortality result across age groups. We divide the observations by whether the group is above or below the median age (36). We find marginally, but insignificantly, bigger effects for older men compared to younger. Since age is such an important factor in determining life expectancy after an AIDS diagnosis, the impact of an early diagnosis should be greater for older men than younger men, ceterus parabus. In addition, it could be the case that older men are more likely to heed medical advice after a positive AIDS diagnosis. 
Figure 8: Heterogeneous Effects of Mortality by Race, Age and Transmission Category
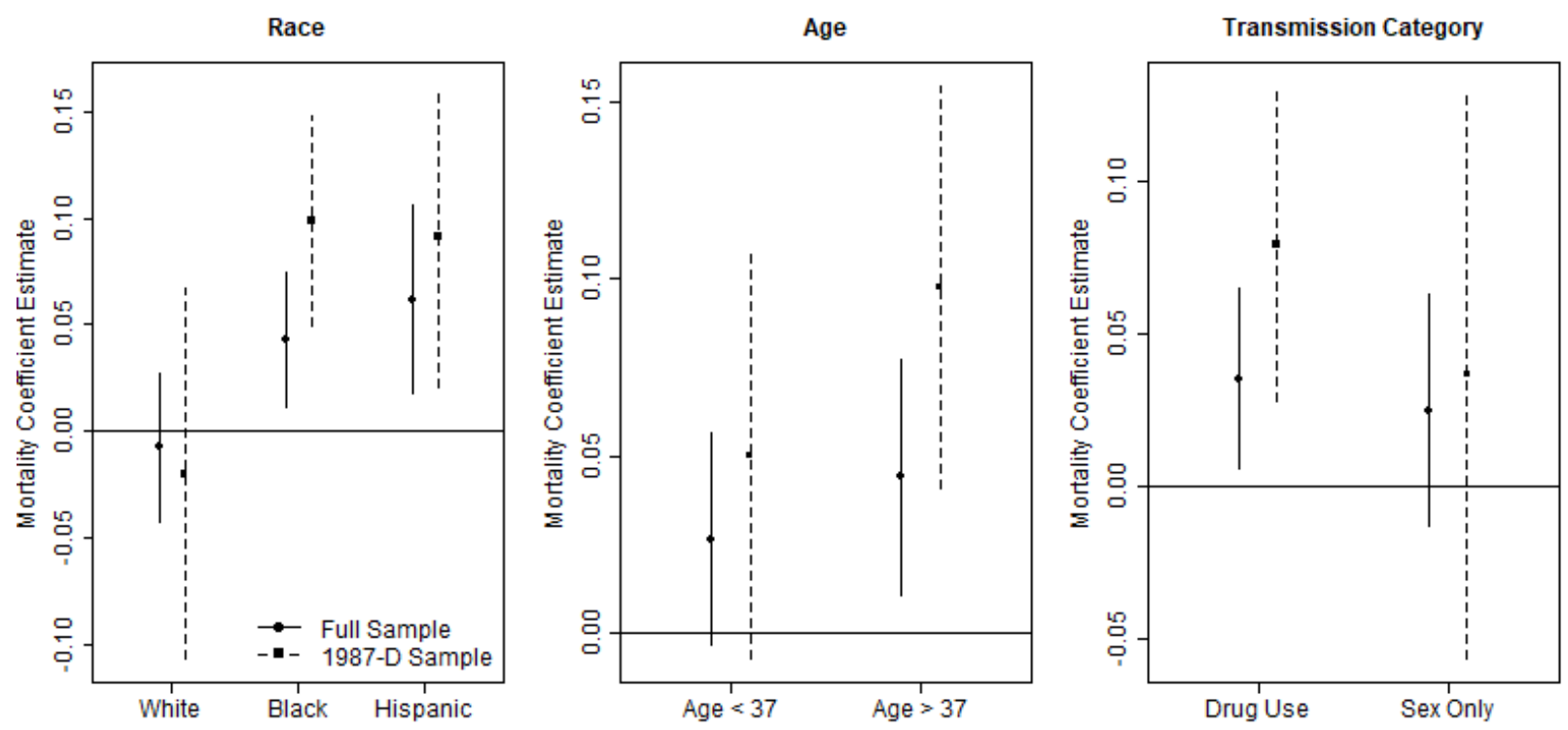

Note: Figure represents heterogeneity of the effect Johnson had on heterosexual men in NBA MSAs. The first panel decomposes the effect by race. The second panel decomposes the effect by age. The final panel decomposes the effect by transmission category. Each panel has both estimates for the full sample as well as estimates for the sample diagnosed using the 1987 Definitive surveillance definition.

Finally, we observe a similar effect by transmission category as we do by age. Drug using men are slightly more likely, compared to non-drug using men, to be alive in the 2000s. However, these men likely have worse immune systems and are more sensitive to an early AIDS diagnosis. In addition, this is the only group where we observe a slightly different rate of substitution towards definitive diagnoses. The coefficient for non-drug using men is larger $(\beta=.06, p=.04)$ compared to the coefficient for drug using men $(\beta=.02, p=.25)$. As mentioned earlier, this is not an easy comparison to make since transmission categories can overlap unlike age or race. In addition, to demonstrate just how "mixed" these groups are, the CDC preformed a condom use study amongst 450 heterosexual drug users in Manhattan, New York in 1991 (CDC, 1992). 77\% of these men reported having a consistent female sexual partner. This is indicative of a severe blending of transmission categories.

\section{Conclusion}

Informational asymmetries between health officials and at-risk populations are fundamental problems in any public health crisis. This issue can be clearly observed during the AIDS pandemic in part due to the unique prejudices towards those who were believed to be solely susceptible to the virus along with the high social and medical costs associated with being identified and labeled as "infected". Public figures disclosing their health status has been documented to change behaviors among at-risk populations, particularly among those groups are similar demographically to the public figure.

Using survey, diagnoses and mortality data, we demonstrate and estimate the causal impact of Earvin 
"Magic" Johnson's announcement of his HIV status towards fixing this asymmetry. This single diagnosis simultaneously increased the public's understanding of, as well as the willingness to be tested for, HIV/AIDS. If we interpret our model as causal, we can make predictions of AIDS diagnoses in a counterfactual world where Johnson is never diagnosed with AIDS. We find that Johnson's announcement increased diagnoses by about 1300 men, with $61 \%$ of these additional diagnoses belonging to heterosexual men. This is a striking number when compared to the fact that the ratio of homosexual to heterosexual diagnoses during this time was close to 3:1.

To put our results in context, evidence collected in 1991 shows that Denver, a large MSA home to an NBA franchise, observed a $200 \%$ increase in HIV/AIDS testing in the 20 days following Johnson's announcement (Cohn et al., 1992) and a 30\% increase in diagnoses over the 13 months following November 1991. If we liberally generalize this observation, in conjunction with our results, we estimate that Johnson's announcement caused an approximate $130 \%$ increase in HIV/AIDS testing, given the estimated average increase in diagnoses (20\%) in other MSAs home to NBA franchises.

Data limitations restrict our ability to observe the full scope and magnitude of Johnson's announcement. First, our outcome measures are only for individuals which had developed AIDS. Given that contracting HIV is a necessary condition for progressing to AIDS, it is likely that the effects that we estimate would be orders of magnitude larger if we were able to observe HIV diagnoses. Second, we are unable to observe the date of death in APIDS. This restricts our analysis to the use of a binary variable reporting whether an individual was alive one decade later. This is not ideal when analyzing patients which suffer from a disease that, once sufficiently progressed, often results in death in less than 1-2 years.

Despite these limitations, recent related research suggests that there may be reason to suspect that these effects were meaningful. For example, Thornton (2008) demonstrates that those who learn of their HIV status are more likely to purchase condoms afterwards, while Godlonton and Thornton (2012) document positive network effects among close neighbors for HIV testing. Given that Johnson's effect on diagnoses is concentrated amongst older Black and Hispanic men in NBA MSAs, we suspect that any spillover effects on attitudes or behavior would also be concentrated in these groups. We leave it to future research to explore these potential spillover effects. ${ }^{26}$

While our estimates are small in magnitude when compared with the overall number of people infected with HIV in the United States, the welfare and economic effects of learning of a diagnosis were likely substantial for those who were impacted. Being diagnosed with a highly advanced case of AIDS would have allowed more people to come to terms with their health, prepare their finances and funerals, and say goodbye to friends and loved ones. For those who caught the syndrome early into its progression, measures taken which minimized one's probability of exposure to opportune infections would have dramatic implications on the probability of surviving until the eventual introduction of HAART. Additionally, the long-run effects stemming from the public's updated knowledge and beliefs allowed people who previously engaged in risky sexual or drug-using behavior to potentially stop the spread of the infection. The diagnoses identified likely had a dramatic effect not only for PWAs, but also for those who would have contracted HIV otherwise.

\footnotetext{
${ }^{26}$ Notably, Thornton (2012) shows that these effects may be detectable only in the short run as there are negligible differences in economic behavior between those who know and do not know their HIV status beyond two years.
} 
"Magic Johnson is an idol," [Dr. Michael] Gottlieb said in an interview with KNBC-TV in Los Angeles. "No one coming down with AIDS except perhaps George Bush would have more impact on this epidemic."

- New York Times, 1991 


\section{References}

Alpert, A., Powell, D., and Pacula, R. L. (2018). Supply-side drug policy in the presence of substitutes: Evidence from the introduction of abuse-deterrent opioids. American Economic Journal: Economic Policy, 10(4):1-35.

Alrababah, A., Marble, W., Mousa, S., and Siegel, A. (2019). Can exposure to celebrities reduce prejudice? The effect of Mohamed Salah on islamophobic behaviors and attitudes. Working Paper.

Beaman, L., Chattopadhyay, R., Duflo, E., Pande, R., and Topalova, P. (2009). Powerful women: Does exposure reduce bias? The Quarterly Journal of Economics, 124(4):1497-1540.

Boisjoly, J., Duncan, G. J., Kremer, M., Levy, D. M., and Eccles, J. (2006). Empathy or antipathy? The impact of diversity. American Economic Review, 96(5):1890-1905.

Casey, M. K., Allen, M., Emmers-Sommer, T., Sahlstein, E., Degooyer, D., Winters, A. M., Wagner, A. E., and Dun, T. (2003). When a celebrity contracts a disease: The example of Earvin "Magic" Johnson's announcement that he was HIV positive. Journal of Health Communication, 8(3):249-265.

CDC (1992). Condom use among male injecting-drug users-New York City, 1987-1990. MMWR. Morbidity and mortality weekly report, 41(34):617-620.

CDC (2020a). Basic statistics. https://www.cdc.gov/hiv/basics/statistics.html.

CDC (2020b). Terms, definitions, and calculations used in CDC HIV surveillance publications. https: //www.cdc.gov/hiv/statistics/surveillance/terms.html.

Centers for Disease Congrol (2020). Stages of HIV infection. https://wwwn.cdc.gov/hivrisk/what_is/ stages_hiv_infection.html.

Choudhary, S. K., Vrisekoop, N., Jansen, C. A., Otto, S. A., Schuitemaker, H., Miedema, F., and Camerini, D. (2007). Low immune activation despite high levels of pathogenic human immunodeficiency virus type 1 results in long-term asymptomatic disease. Journal of Virology, 81(16):8838-8842.

Cohn, D. L., Miller, L. A., Yamaguchi, K. J., and Douglas, J. M. (1992). Denver's increase in hiv counseling after Magic Johnson's HIV disclosure. American Journal of Public Health, 82(12):1692-1692.

Cram, P., Fendrick, A. M., Inadomi, J., Cowen, M. E., Carpenter, D., and Vijan, S. (2003). The impact of a celebrity promotional campaign on the use of colon cancer screening: The Katie Couric effect. Archives of Internal Medicine, 163(13):1601-1605.

Dillender, M. (2021). Evidence and lessons on the health impacts of public health funding from the fight against HIV/AIDS. Technical report, National Bureau of Economic Research.

Evans, D. G. R., Barwell, J., Eccles, D. M., Collins, A., Izatt, L., Jacobs, C., Donaldson, A., Brady, A. F., Cuthbert, A., Harrison, R., et al. (2014). The angelina jolie effect: how high celebrity profile can have a major impact on provision of cancer related services. Breast Cancer Research, 16(5):1-6.

Ewings, F. M., Bhaskaran, K., McLean, K., Hawkins, D., Fisher, M., Fidler, S., Gilson, R., Nock, D., Brettle, R., Johnson, M., et al. (2008). Survival following hiv infection of a cohort followed up from seroconversion in the uk. Aids, 22(1):89-95.

Fernández, R. and Parsa, S. (2021). Gay politics goes mainstream: Democrats, Republicans, and same-sex relationships. Technical report, National Bureau of Economic Research.

Fernández, R., Parsa, S., and Viarengo, M. (2019). Coming out in America: AIDS, politics, and cultural change. Technical report, National Bureau of Economic Research.

Fischl, M. A., Richman, D. D., Grieco, M. H., Gottlieb, M. S., Volberding, P. A., Laskin, O. L., Leedom, J. M., Groopman, J. E., Mildvan, D., Schooley, R. T., et al. (1987). The efficacy of azidothymidine (AZT) in the treatment of patients with AIDS and AIDS-related complex. New England Journal of Medicine, 
317(4):185-191.

Flora, J. A., Schooler, C., Mays, V. M., and Cochran, S. D. (1996). Exploring a model of symbolic social communication: The case of 'Magic' Johnson. Journal of Health Psychology, 1(3):353-366.

Fryer Jr, R. G., Heaton, P. S., Levitt, S. D., and Murphy, K. M. (2013). Measuring crack cocaine and its impact. Economic Inquiry, 51(3):1651-1681.

Ganguly, A. and Tasoff, J. (2017). Fantasy and dread: The demand for information and the consumption utility of the future. Management Science, 63(12):4037-4060.

Garcia, S. A. B. and Guzman, N. (2018). Acquired immune deficiency syndrome (AIDS) CD4+ count. Europe PMC.

Garthwaite, C. and Moore, T. J. (2013). Can celebrity endorsements affect political outcomes? Evidence from the 2008 US Democratic presidential primary. The Journal of Law, Economics, 85 Organization, $29(2): 355-384$.

Garthwaite, C. L. (2014). Demand spillovers, combative advertising, and celebrity endorsements. American Economic Journal: Applied Economics, 6(2):76-104.

Garz, M. and Pagels, V. (2018). Cautionary tales: Celebrities, the news media, and participation in tax amnesties. Journal of Economic Behavior \& Organization, 155:288-300.

Godlonton, S. and Thornton, R. (2012). Peer effects in learning HIV results. Journal of Development Economics, 97(1):118-129.

Godlonton, S. and Thornton, R. L. (2013). Learning from others' HIV testing: Updating beliefs and responding to risk. American Economic Review, 103(3):439-44.

Golman, R., Hagmann, D., and Loewenstein, G. (2017). Information avoidance. Journal of Economic Literature, 55(1):96-135.

Group, I. S. S. (2015). Initiation of antiretroviral therapy in early asymptomatic HIV infection. New England Journal of Medicine, 373(9):795-807.

Hamilton, J. D., Hartigan, P. M., Simberkoff, M. S., Day, P. L., Diamond, G. R., Dickinson, G. M., Drusano, G. L., Egorin, M. J., George, W. L., Gordin, F. M., et al. (1992). A controlled trial of early versus late treatment with zidovudine in symptomatic human immunodeficiency virus infection. New England Journal of Medicine, 326(7):437-443.

Hatano, H., Yukl, S. A., Ferre, A. L., Graf, E. H., Somsouk, M., Sinclair, E., Abdel-Mohsen, M., Liegler, T., Harvill, K., Hoh, R., et al. (2013). Prospective antiretroviral treatment of asymptomatic, HIV-1 infected controllers. PLOS Pathogens, 9(10).

Herek, G. M. and Capitanio, J. P. (1997). AIDS stigma and contact with persons with AIDS: Effects of direct and vicarious contact. 1. Journal of Applied Social Psychology, 27(1):1-36.

Herek, G. M. and Capitanio, J. P. (1998). Symbolic prejudice or fear of infection? A functional analysis of AIDS-related stigma among heterosexual adults. Basic and applied social psychology, 20(3):230-241.

Herek, G. M., Capitanio, J. P., and Widaman, K. F. (2002). HIV-related stigma and knowledge in the United States: Prevalence and trends, 1991-1999. American Journal of Public Health, 92(3):371-377.

Herek, G. M., Widaman, K. F., and Capitanio, J. P. (2005). When sex equals AIDS: Symbolic stigma and heterosexual adults' inaccurate beliefs about sexual transmission of AIDS. Social Problems, 52(1):15-37.

Hingson, R., Strunin, L., Craven, D. E., Mofenson, L., Mangione, T., Berlin, B., Amaro, H., and Lamb, G. A. (1989). Survey of aids knowledge and behavior changes among massachusetts adults. Preventive Medicine, 18(6):806-816.

Hogg, R. S., Strathdee, S. A., Craib, K., O’Shaughnessy, M. V., Montaner, J., and Schechter, M. T. (1997). 
Modelling the impact of HIV disease on mortality in gay and bisexual men. International Journal of Epidemiology, 26(3):657-661.

Kalichman, S. C. and Hunter, T. L. (1992). The disclosure of celebrity HIV infection: Its effects on public attitudes. American Journal of Public Health, 82(10):1374-1376.

Kelly, J. A., St Lawrence, J. S., Stevenson, L. Y., Hauth, A. C., Kalichman, S. C., Diaz, Y. E., Brasfield, T. L., Koob, J. J., and Morgan, M. G. (1992). Community AIDS/HIV risk reduction: the effects of endorsements by popular people in three cities. American Journal of Public Health, 82(11):1483-1489.

Köszegi, B. (2003). Health anxiety and patient behavior. Journal of Health Economics, 22(6):1073-1084.

Larson, R. J., Woloshin, S., Schwartz, L. M., and Welch, H. G. (2005). Celebrity endorsements of cancer screening. Journal of the National Cancer Institute, 97(9):693-695.

Lee, L. M., Karon, J. M., Selik, R., Neal, J. J., Fleming, P. L., et al. (2001). Survival after AIDS diagnosis in adolescents and adults during the treatment era, United States, 1984-1997. JAMA-Journal of the American Medical Association-International Edition, 285(10):1308-1315.

Lemp, G. F., Payne, S. F., Neal, D., Temelso, T., and Rutherford, G. W. (1990). Survival trends for patients with AIDS. Journal of the American Medical Association, 263(3):402-406.

Metcalfe, D., Price, C., and Powell, J. (2011). Media coverage and public reaction to a celebrity cancer diagnosis. Journal of public health, 33(1):80-85.

Nattinger, A. B., Hoffmann, R. G., Howell-Pelz, A., and Goodwin, J. S. (1998). Effect of Nancy Reagan's mastectomy on choice of surgery for breast cancer by US women. Journal of the American Medical Association, 279(10):762-766.

Oster, E., Shoulson, I., and Dorsey, E. (2013). Optimal expectations and limited medical testing: Evidence from huntington disease. American Economic Review, 103(2):804-30.

Padian, N., Shiboskl, S., and Jewell, N. (1990). The effect of number of exposures on the risk of heterosexual HIV transmission. Journal of Infectious Diseases, 161(5):883-887.

Panel on Antiretroviral Guidelines for Adults and Adolescents (2019). Guidelines for the use of antiretroviral agents in adults and adolescents with HIV.

Peterman, T. A., Stoneburner, R. L., Allen, J. R., Jaffe, H. W., and Curran, J. W. (1988). Risk of human immunodeficiency virus transmission from heterosexual adults with transfusion-associated infections. JAMA, 259(1):55-58.

Petro, A. M. (2015). After the wrath of God: AIDS, sexuality, and American religion. Oxford University Press, USA.

Pollock III, P. H. (1994). Issues, values, and critical moments: Did "Magic" Johnson transform public opinion on AIDS? American Journal of Political Science, pages 426-446.

Rosenblum, L., Buehler, J. W., Morgan, M. W., Costa, S., Hidalgo, J., Holmes, R., Lieb, L., Shields, A., and Whyte, B. M. (1992). The completeness of AIDS case reporting, 1988: A multisite collaborative surveillance project. American journal of public health, 82(11):1495-1499.

Sax, P. E., Bartlett, J., and Bloom, A. (2019). Acute and early HIV infection: Clinical manifestations and diagnosis. Up To Date.

Shayne, V. T. and Kaplan, B. J. (1991). Double victims: Poor women and AIDS. Women EJ Health, $17(1): 21-37$.

Stokes, J. P. and Peterson, J. L. (1998). Homophobia, self-esteem, and risk for HIV among African American men who have sex with men. AIDS Education and Prevention.

Thornton, R. L. (2008). The demand for, and impact of, learning HIV status. American Economic Review, 
98(5):1829-63.

Thornton, R. L. (2012). HIV testing, subjective beliefs and economic behavior. Journal of Development Economics, 99(2):300-313.

Valdiserri, R. O. (2002). HIV/AIDS stigma: An impediment to public health.

Walther, C. S. and Poston Jr, D. L. (2004). Patterns of gay and lesbian partnering in the larger metropolitan areas of the United States. Journal of Sex Research, 41(2):201-214.

Wang, T. (2021). Media, pulpit, and populist persuasion: Evidence from Father Coughlin. Forthcoming: American Economic Review. 


\section{A Appendix}

\section{A.1 Commonly Used Abbreviations}

Table A.1: Commonly Used Abbreviations

\begin{tabular}{c|l}
\hline Abbreviation & Definition \\
\hline $4 \mathrm{H}$ & Haitians, Homosexuals, Hemophiliacs, and Heroin users \\
AIDS & Acquired Immunodeficiency Syndrome \\
APIDS & AIDS Public Information Data \\
AZT & Azidothymidine \\
CARE Act & Comprehensive AIDS Resources Emergency Act \\
CDC & Centers for Disease Control and Prevention \\
DMA & Designated Market Area \\
FDA & Food and Drug Administration \\
GRID & Gay-Related Immune Deficiency \\
HAART & Highly Active Antiretroviral Therapy \\
HIV & Human Immunodeficiency Virus \\
MSA & Metropolitan Statistical Area \\
NBA & National Basketball Association \\
NCHS & National Center for Health Statistics \\
NHIS & National Health Interview Survey \\
PWA & Person(s) with AIDS \\
\hline
\end{tabular}

Note: This table defines some commonly used abbreviations throughout the study as a reference.

\section{A.2 AIDS Diagnoses vs HIV Diagnoses}

This study leverages AIDS diagnoses rather than HIV. While this was brought about due to data constraints, as the CDC only reported AIDS diagnoses, there are many reasons why we believe these do not substantively limit the interpretation of our results. In fact, employing AIDS rather than HIV diagnoses implies that our results could be significantly downwardly biased due to the fact that it is much more likely that one will be diagnosed with HIV than its more progressed form in AIDS.

An AIDS diagnosis in the early 1990s was an almost certain death sentence for many, particularly the poor (Lemp et al., 1990; Shayne and Kaplan, 1991). However, it was not an instant death sentence due to the nature of HIV/AIDS which, among many other things, dramatically weakens the immune system leaving one vulnerable to minor external health shocks (Hogg et al., 1997). The definition, which differentiates patients who are living with HIV from those who have developed AIDS, is typically much more clear in the eyes of physicians than it is for patients due to the fact that PWAs typically do not notice any substantially change in well-being when moving beyond the threshold for having developed AIDS. ${ }^{27}$ Though patients' lives are at great risk following any health shock or sickness, PWAs can be largely asymptomatic with very low CD4 levels as a result of how the virus typically progresses. For example, patients experience the majority of their

\footnotetext{
${ }^{27}$ One requires 200 or less CD4+ T cells (compared to the $1000 \mathrm{avg}$ ) and/or for one's CD4+ T cells to consist of less than $14 \%$ of all lymphocytic cells to be diagnosed with AIDS (CDC, 2020b; Garcia and Guzman, 2018).
} 
HIV-related symptoms during the first 2-4 weeks after infection. ${ }^{28,29}$

The fact that potentially significant portions of the population with T-cell counts low enough to have developed AIDS can live largely asymptomatically bolsters our identification. Further, the Panel on Antiretroviral Guidelines for Adults and Adolescents (2019) notes that clinicians may fail to recognize HIV infection in patients who are symptomatic due to the fact that early symptoms are often very similar to those of many other viral infections such as influenza and infectious mononucleosis. Even today many remain unaware of their HIV+/AIDS status. The CDC estimated in 2016 that 1.1 million people over the age of 13 lived with an HIV infection, of whom $14 \%$ were completely unaware (CDC, 2020a).

\section{A.3 Identification of NBA MSAs}

Some MSAs in the sample are easily defined as having an NBA team. For example, MSAs like Atlanta (Hawks), Boston (Celtics), and Chicago (Bulls) have been home to an NBA team since the 1960s. However, there are a handful of MSAs that gained a team either by way of relocation or via league expansion. These MSAs, and the year their team moved to them, are displayed in Table A.2. Each of these MSAs gained their NBA team before Magic Johnson's announcement. All of our results are qualitatively similar when either dropping these from the sample, or denoting them as their own group (neither "control" nor "NBA").

Table A.2: MSAs That Obtained an NBA Team After 1985

\begin{tabular}{cccc}
\hline MSA & State & Team & Year Introduced \\
\hline Sacramento & CA & Kings & 1986 \\
Charlotte & NC & Hornets / Bobcats & 1989 \\
Miami & FL & Heat & 1989 \\
Minneapolis-St Paul & MN & Timberwolves & 1990 \\
Orlando & FL & Magic & 1990 \\
\hline
\end{tabular}

Note: Table presents MSAs that obtained NBA teams during the sample period.

\section{A.4 Testing Other Cutoffs}

While the results from the NHIS survey questions gives us confidence that a change occurred in November 1991, we want to ensure that diagnoses were not trending upwards before or after our identification of Johnson's announcement. In order to do this, we rerun the model identifying other months in the sample as a placebo for Johnson's announcement. We collect the parameter estimates and standard errors in each regression and plot them in Figure A.1. Ideally, we would observe only a single statistically significant point

\footnotetext{
${ }^{28}$ The CDC describes the three stages of disease that one typically progresses through when people get HIV (without modern treatment). The first stage is defined as an "acute HIV" which occurs within 2-4 weeks after infection. One typically has a large amount of the virus in one's blood during this stage. Further, one is very contagious during this period and will likely experience a flu-like illness which can persist for a few weeks as a result. The transition to the second stage is formally referred to as clinical latency, where HIV is still active but the rate at which the virus reproduces slows dramatically. This amount of time largely varies from patient to patient and is a function of the amount of one's CD4 cells pre-infection. As a result, one may not have any symptoms during this time. It is for this reason that this stage is occasionally referred to as asymptomatic HIV. The third stage is when one's HIV infection has eroded away sufficiently enough CD4 cells in order to progress into AIDS (Centers for Disease Congrol, 2020). From here, the degree to which one presents symptoms is a function of how quickly their viral load and CD4 count change inversely. For example, if two patients progress into stage 3 at the same time, the patient who has spent less time in stages 1 and 2 of infection is more likely to have clear symptoms.

${ }^{29}$ The subject of patients living with, but failing to develop HIV/AIDS-related symptoms has a large medical literature dedicated to it (Choudhary et al., 2007; Hatano et al., 2013; Group, 2015; Sax et al., 2019).
} 
above the dashed zero line corresponding to Johnson's announcement. However, we observe a set of three points, and the dates of which are September, October and November 1991. Given these dates are bunched around Johnson's announcement, we consider this evidence of a clean break. We also demarcate two other potentially important dates. The dashed vertical lines, from left to right, represent Ryan White's death and the date that the CARE act was passed.

Figure A.1: Testing Different DID Cutoffs

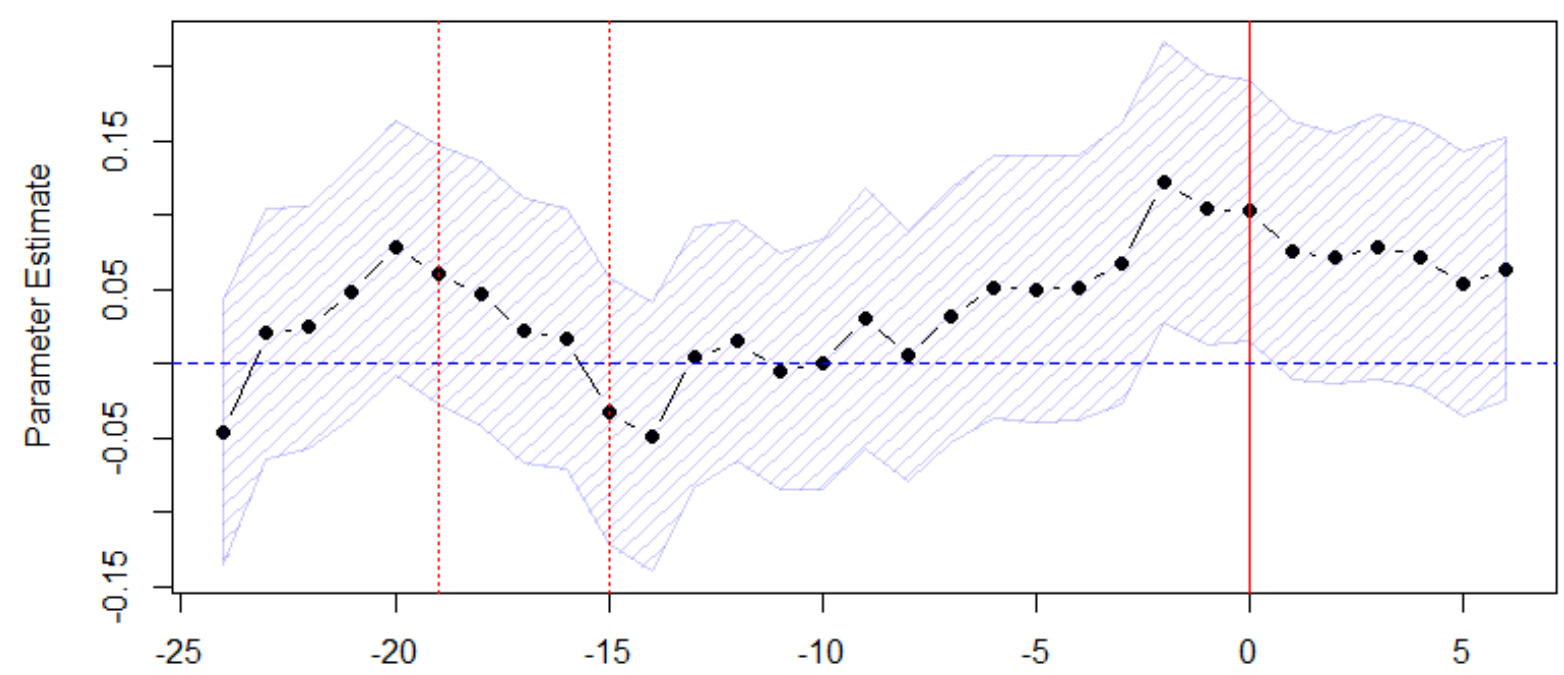

Note: We estimate column (1) in Table 6 and imagine the Magic Johnson's announcement came in different months. We collect the parameter estimates and standard errors and plot them here. The solid vertical line represents the month of Johnson's announcement and the dashed vertical line marks White's death.

Finally, in Figure A.2 we again test our temporal identification by redoing the placebo test in Figure A.1, but this time for $\delta_{2}$, or the parameter estimate for NBA x Magic x Heterosexual. This plot closely resembles Figure A.2 in that there is only one spot that appears to be most significant.

\section{A.5 Varying Samples}

\section{A.5.1 Diagnoses}

To examine the sensitivity of our results to different specifications, definitions, and sample, we first modify the regressions that test for changes in rates of diagnoses. We reestimate the model in column 4 of Table 6 for each. First, we include all MSAs except San Juan and Honolulu and present the results in column 1 of Table A.3. Next, we vary which groups are included from Table 3. For this, we first use only those men who have sex-based risk categories. In the original sample, $8 \%$ of observations are equal to zero. In the sex-only sample, this increases to $32 \%$ ( $65 \%$ for heterosexual communities, $1 \%$ for homosexual). Second, we include women in addition to the original categories. Like the CDC, we have to assume all women in the sample are heterosexual. 
Figure A.2: Testing Different Triple Difference Cutoffs

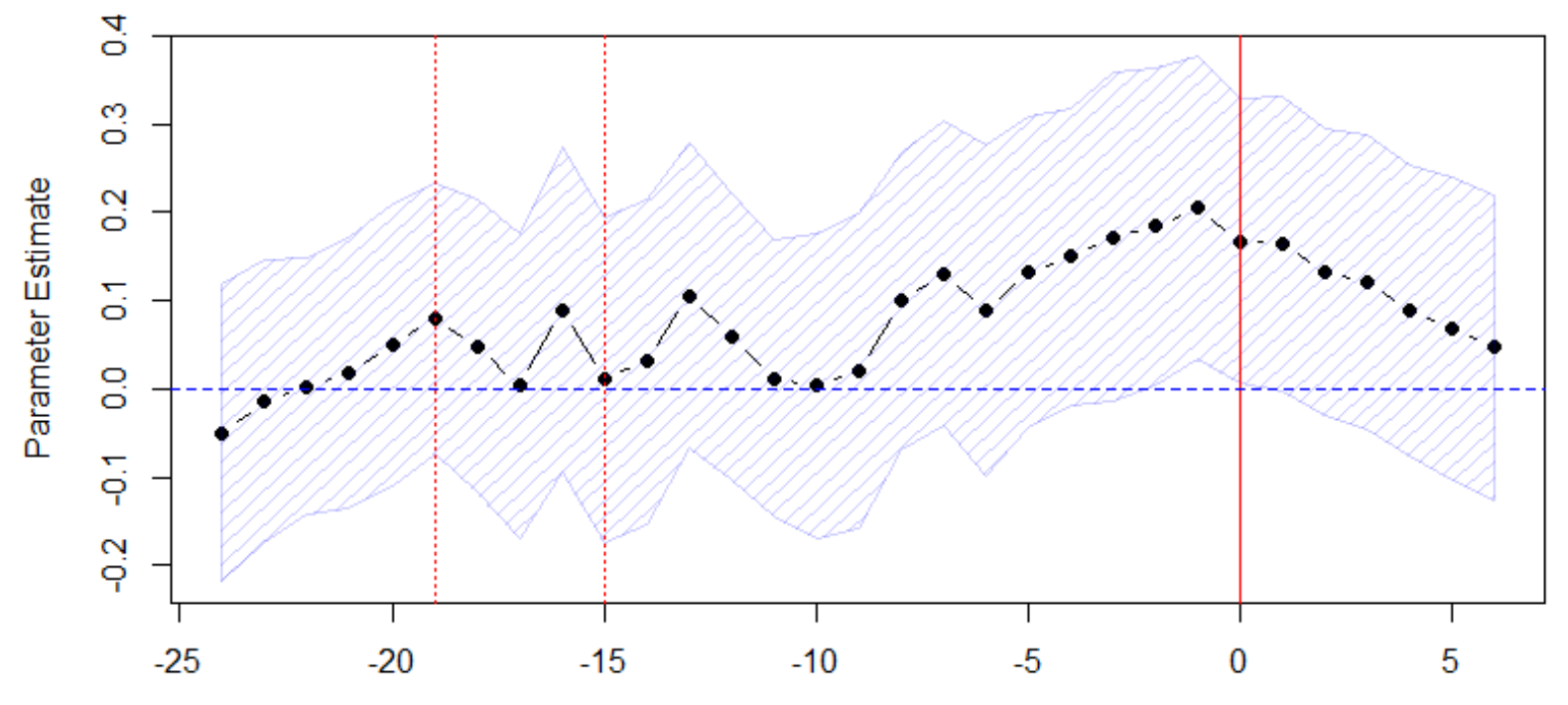

Note: We estimate column (1) in Table 6 and imagine the Magic Johnson's announcement came in different months. We collect the parameter estimates and standard errors and plot them here. The solid vertical line represents the month of Johnson's announcement and the dashed vertical line marks White's death.

Table A.3: AIDS Diagnoses Following Magic Johnson - Alternates

\begin{tabular}{lccc}
\hline & \multicolumn{3}{c}{ sinh $^{-1}$ (Diagnoses) } \\
\hline Magic & -0.030 & -0.059 & -0.040 \\
Magic x Heterosexual & $(0.035)$ & $(0.049)$ & $(0.049)$ \\
& $0.125^{* * *}$ & $0.128^{*}$ & 0.107 \\
Magic x NBA & $(0.048)$ & $(0.066)$ & $(0.075)$ \\
& -0.025 & 0.002 & -0.008 \\
Magic x Heterosexual x NBA & $(0.055)$ & $(0.062)$ & $(0.061)$ \\
& $0.170^{* *}$ & 0.055 & 0.121 \\
Num.Obs. & $(0.074)$ & $(0.096)$ & $(0.090)$ \\
Sample & 11,136 & 7,296 & 7,296 \\
R2 & All MSAs & Only Sex & Incl. Women \\
N $^{*} p<0.1^{* *} p<0.05, * * *$ & 0.874 & 0.908 & 0.851 \\
\hline
\end{tabular}

$* \mathrm{p}<0.1, * * \mathrm{p}<0.05,{ }^{* * *} \mathrm{p}<0.01$

Note: These regressions estimate the effect of Magic Johnson's announcement on the number of AIDS diagnoses. Each regression contains fixed effects for MSA-bySexuality and month of diagnosis. In addition, specific MSA-by-Sexuality trends are also controlled for. Standard errors are clustered at the MSA-by-Sexuality level. 


\section{A.5.2 Composition \& Mortality}

Similarly, we examine how including more MSAs and women into our analyses change the results. We do not test for differences in Sex-only definitions since we do something very similar in the section detailing the heterogeneous effects. Instead of presenting more tables, we replicate Figures 6 and 7 with additional MSAs and women.

\section{A.6 Google Trends}

An analysis of Google Trends data shows that the NBA is much more popular in areas with NBA teams than in areas without NBA teams, but with other pro-sports teams (denoted non-NBA). The results of this analysis is very similar when looking at NBA DMAs compared to all other DMAs and not just others with professional sports.

Google Trends data come with a few important caveats that are crucial for proper interpretation. First, the finest level of spatial granularity is the Nielsen's Designated Market Areas (DMAs). These are similar to MSAs but more focused on television markets than populations. Each DMA in the sample is augmented with an indicator variable equal to 1 if the DMA contains an NBA team. This is how we differentiate between the DMAs. Second, when extracting data from Google Trends, the data are scaled such that the maximum for any query is always 100. For example, if a query is made to extract the popularity of the NBA in DMA $A$, the maximum popularity will be equal to 100 . Then, when querying DMA $B$, the maximum will again be equal to 100. Unfortunately, these 100s are not necessarily equal. Rather, one must query both DMA $A$ and DMA $B$ at the same time in order to accurately compare the relative popularity. Therefore, search term popularity is always relative to some baseline DMA. The same approach must be used for multiple search terms. Finally, Google Trends slightly changed their methodology in 2011 and 2016 which is denoted in Figure A.5 by the dashed vertical lines.

We query Google for search popularity of "NBA" and "Sports" for all DMAs for 2004 through 2020. Once the search popularity numbers for each term are adjusted, they are averaged by month and whether the DMA contains an NBA team. Then, we calculate the ratio of each terms popularity in NBA DMAs vs non-NBA DMAs and subtract 1 . Therefore, a value of .25 would suggest the term is $25 \%$ more popular in NBA markets compared to non-NBA. Figure A.5 displays the results of analyzing the popularity of "NBA" and "Sports". The values for the term "Sports" hover around and just below 0. This signifies that "Sports" is just as, if not more, popular in non-NBA DMAs as NBA DMAs. However, the term "NBA" is consistently over $20 \%$ more popular in NBA DMAs. Remember, these are DMAs with other professional sports teams, which is likely the most comparable group to DMAs with NBA teams.

While these results are for a time period 10-20 years after Johnson's announcement, we assert that this relationship must have been similar during the late 1980s and early 1990s. There is no way to empirically test this, but with the introduction of the internet and increasing popularity of the NBA, it would be surprising if the differences found were not even larger during the time of Johnson's announcement.

\section{A.7 Additional Information on APIDS}

APIDS is an extremely comprehensive dataset on AIDS diagnoses in the first two decades of the epidemic. Table A.4 displays some of the key variables used in this study and their definitions. 
Figure A.3: Robustness - all MSAs
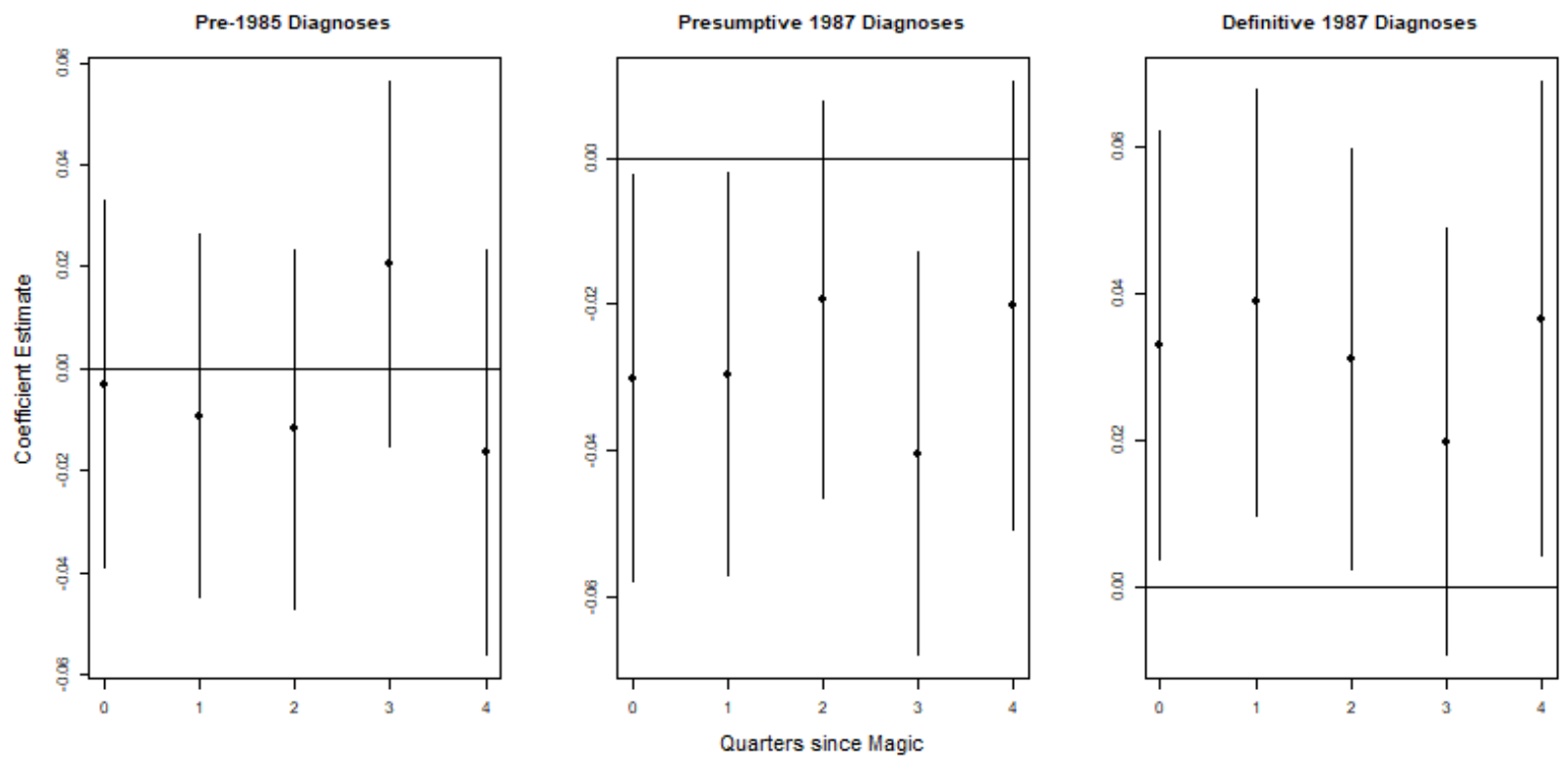

(a) Changes in Composition of Surveillance Definition Over Time
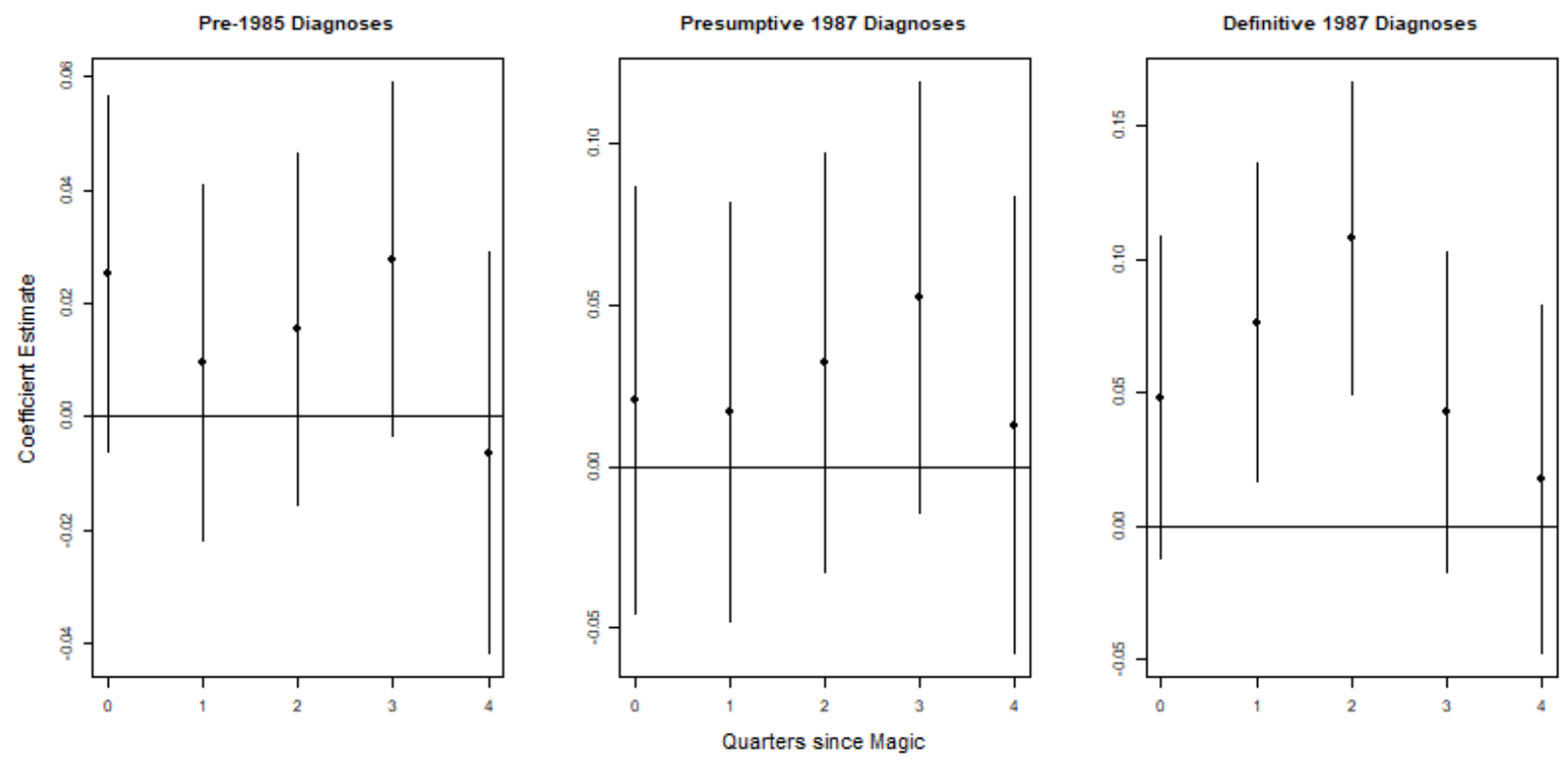

(b) Changes in Mortality by Definition Over Time

Note: Figure presents similar results as in Figures 6 and 7, except recalculated with all MSAs (besides San Juan and Honolulu) in APIDS. 
Figure A.4: Robustness - Incl. Women
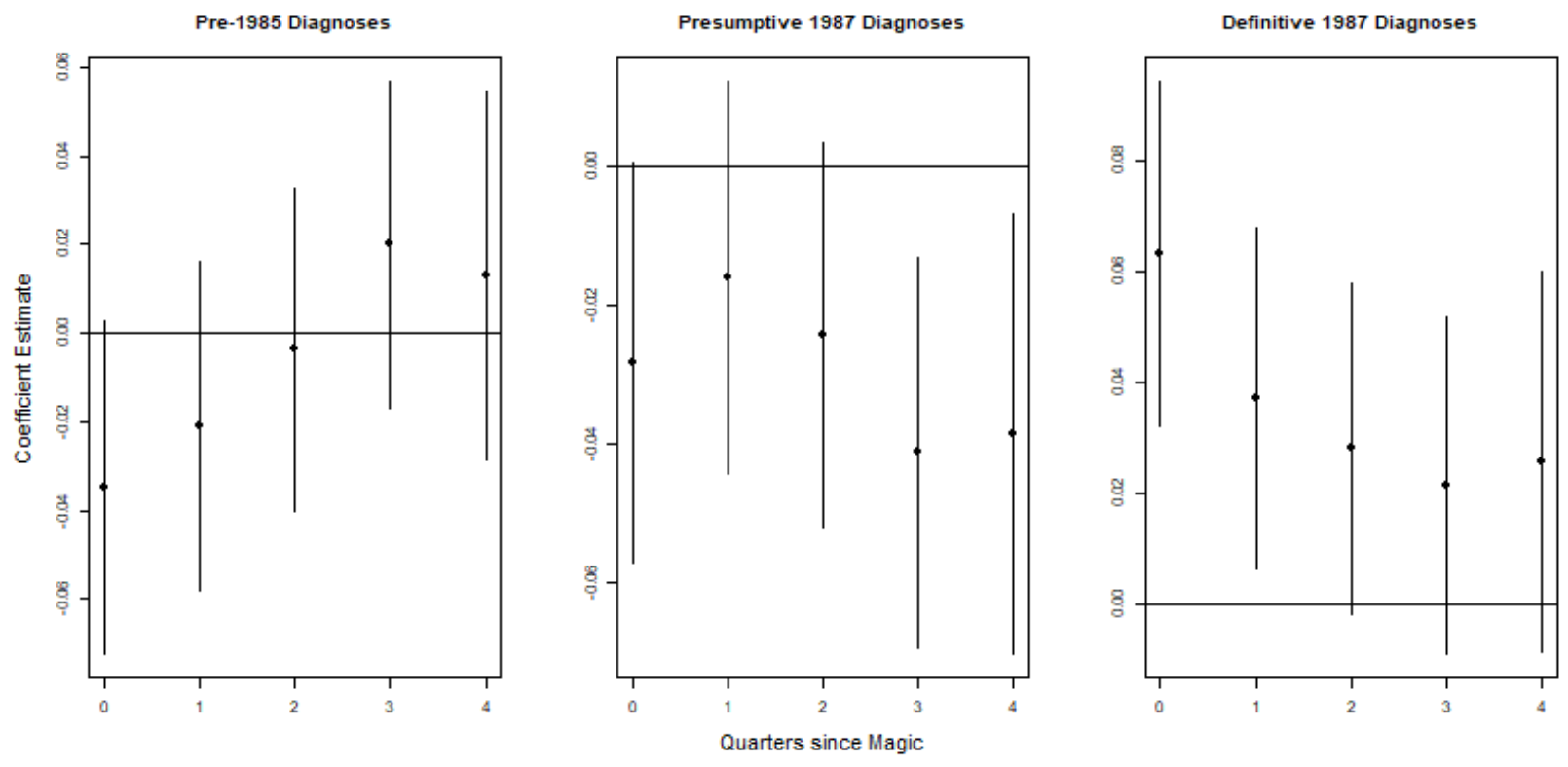

(a) Changes in Composition of Surveillance Definition Over Time
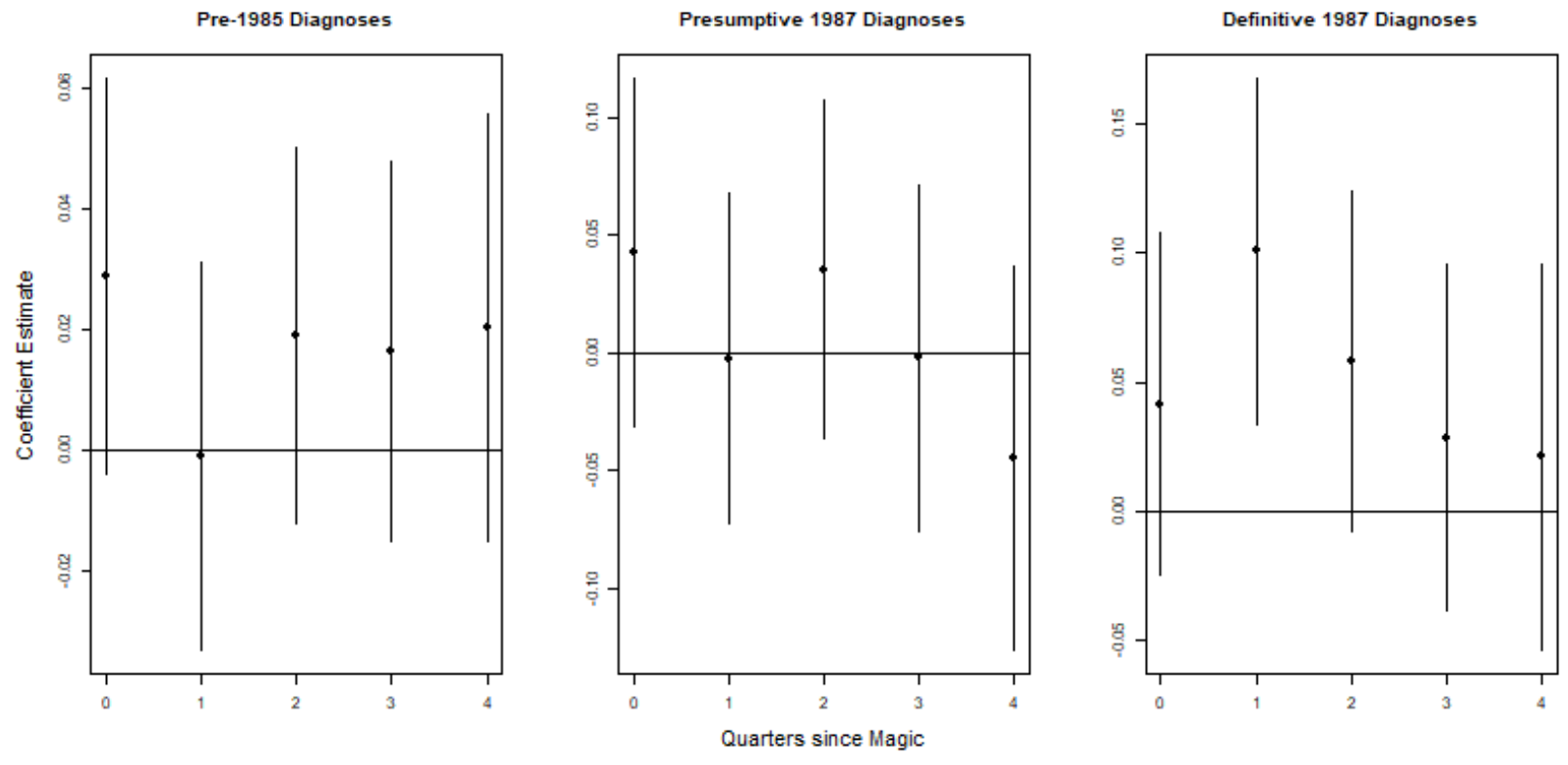

(b) Changes in Mortality by Definition Over Time

Note: Figure presents similar results as in Figures 6 and 7, except recalculated by including women into the heterosexual sex group. 
Figure A.5: NBA Popularity in NBA MSAs

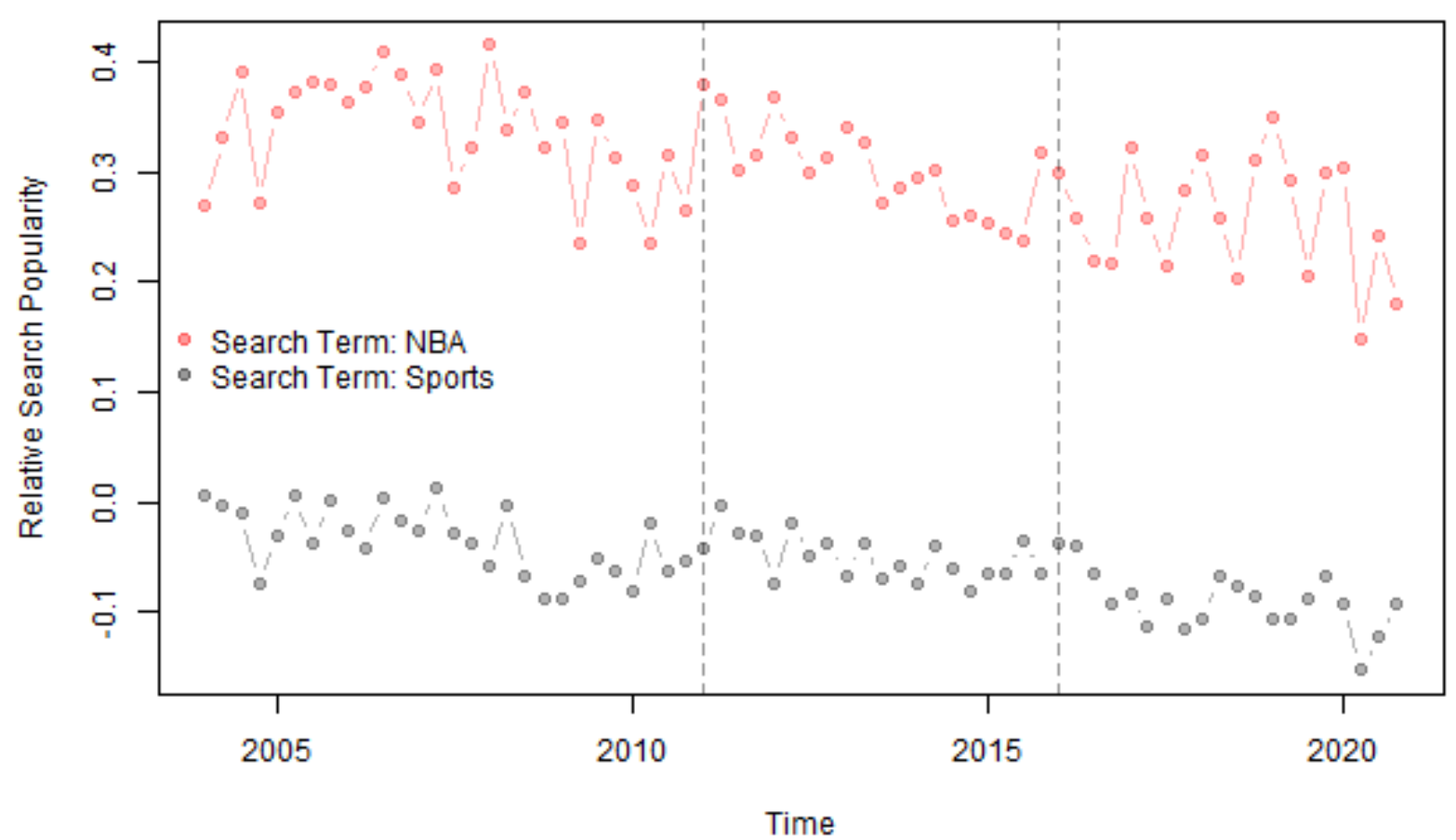

Note: Figure represents the relative search popularity of "NBA" and "Sports" in DMAs with NBA teams compared to those without an NBA team. The y-axis represents the additional popularity of each search term on average in NBA DMAs.

Table A.4: Definitions of Key APIDS Variables

\begin{tabular}{l|l|l} 
APIDS Variable & Definition & Notes \\
\hline GENDER & Biological Gender and Sexuality & $\begin{array}{l}\text { The CDC provides four options: homosexual } \\
\text { male, bisexual male, heterosexual male, and } \\
\text { female. }\end{array}$ \\
\hline TRANSCAT & Transmission Category & $\begin{array}{l}\text { Main behavioral risk factor likely responsible } \\
\text { for how AIDS was obtained. }\end{array}$ \\
\hline CATEG & AIDS Surveillance Definition & $\begin{array}{l}\text { There are 7 possible surveillance definitions a } \\
\text { patient can qualify for. }\end{array}$ \\
\hline DEATH & Vital Status & $\begin{array}{l}\text { This is a binary variable telling whether the } \\
\text { CDC has received a notice of death for the } \\
\text { patient. }\end{array}$ \\
\hline DXDATE & Diagnosis Date & Month and year of diagnosis. \\
\hline REPDATE & Report Date & Month and year of CDC report. \\
\hline
\end{tabular}

Note: Table displays definitions and notes about key APIDS variables. 


\section{A.7.1 Mortality}

Figure A.6 displays the empirical probability of surviving until 2000 by diagnosis month for the four most popular surveillance definitions. In addition, we include vertical lines that represent the beginning and end of the study sample and Johnson's announcement. After the sample period ends, there appears to be a kink, which coincides with the introduction of HAART, or the Triple Cocktail. This was a novel and effective treatment for HIV/AIDS, so it is unsurprising to observe this kink. While most of the time series is positively sloped, indicating an intuitive increasing probability of survival for each successive month, there is also a peculiar negative slope for the 1993 definition before 1993, and negative slope for the 1987 definitions before 1987. Diagnoses of a certain surveillance definition before the definition way in use are retroactive AIDS diagnoses. Therefore, if someone was tested for HIV/AIDS in 1992 but only qualified for the 1993 definition, they would have been told they were AIDS negative. These negative slopes are due to difficulties in following up with these retroactive cases.

Figure A.6: AIDS Definition Frequencies \& Survival Rates by Diagnosis Month

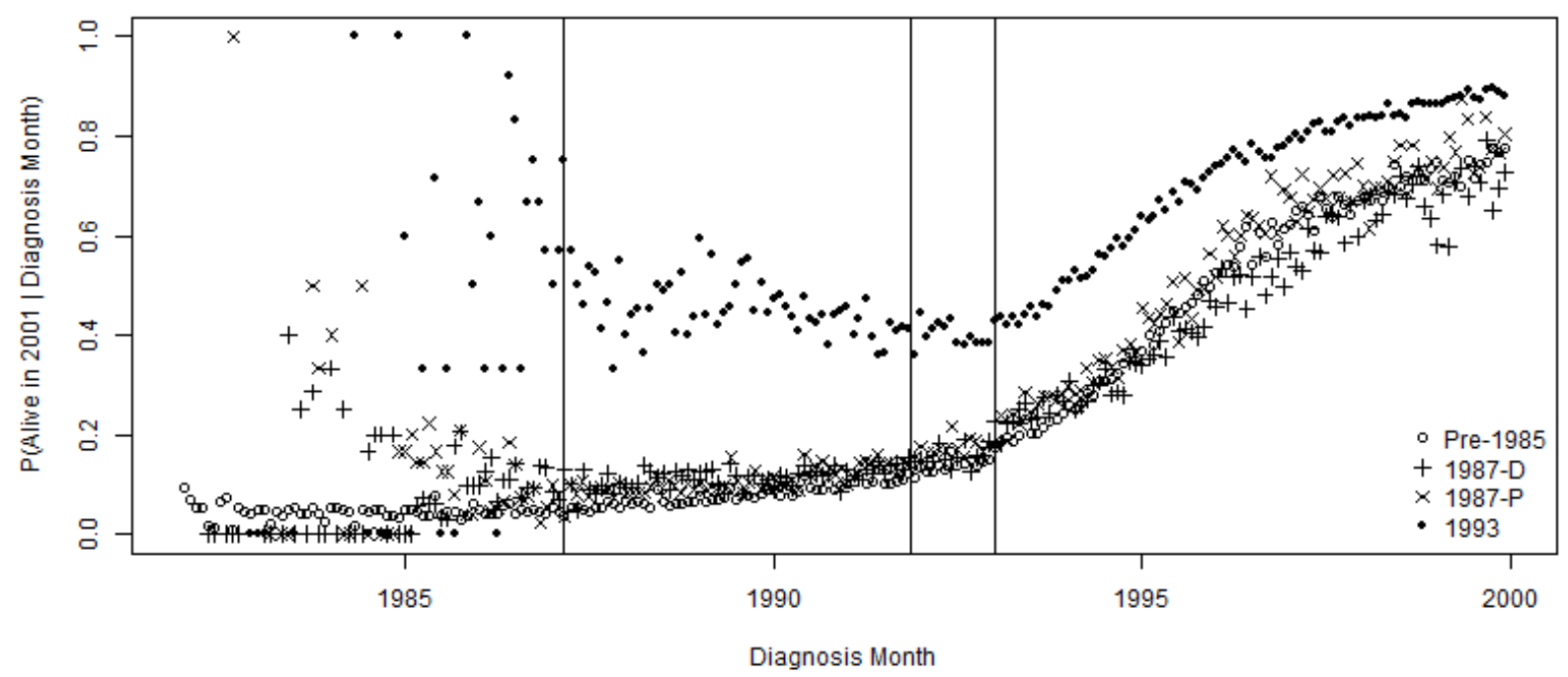

Note: This figure represents the survival curve by month of diagnosis. All diagnoses after December 2000 are listed as alive in the 2002 version of APIDS.

\section{A.7.2 Report Delay}

Aside from the cases that are diagnosed once a new surveillance definition is implemented, there is also the case of report delay. As mentioned previously, there are two important dates for each entry in APIDS. First, the diagnosis date tells when the individual had their test done. Second, the report date tells when the CDC finds out about the positive test. Since the CDC is supposed to follow up with these cases, the report delay is crucial to obtaining accurate information. As time elapses without the CDC being informed, it becomes increasingly difficult for the CDC to follow up. In fact, there is an extremely strong correlation between report delay and probability of the CDC obtaining a notification of death. This is shown in Figure A.7. The left panel displays the overall relationship while the right panel is broken down by diagnosis category. We 
Figure A.7: Probability of Survival by Reporting Delay
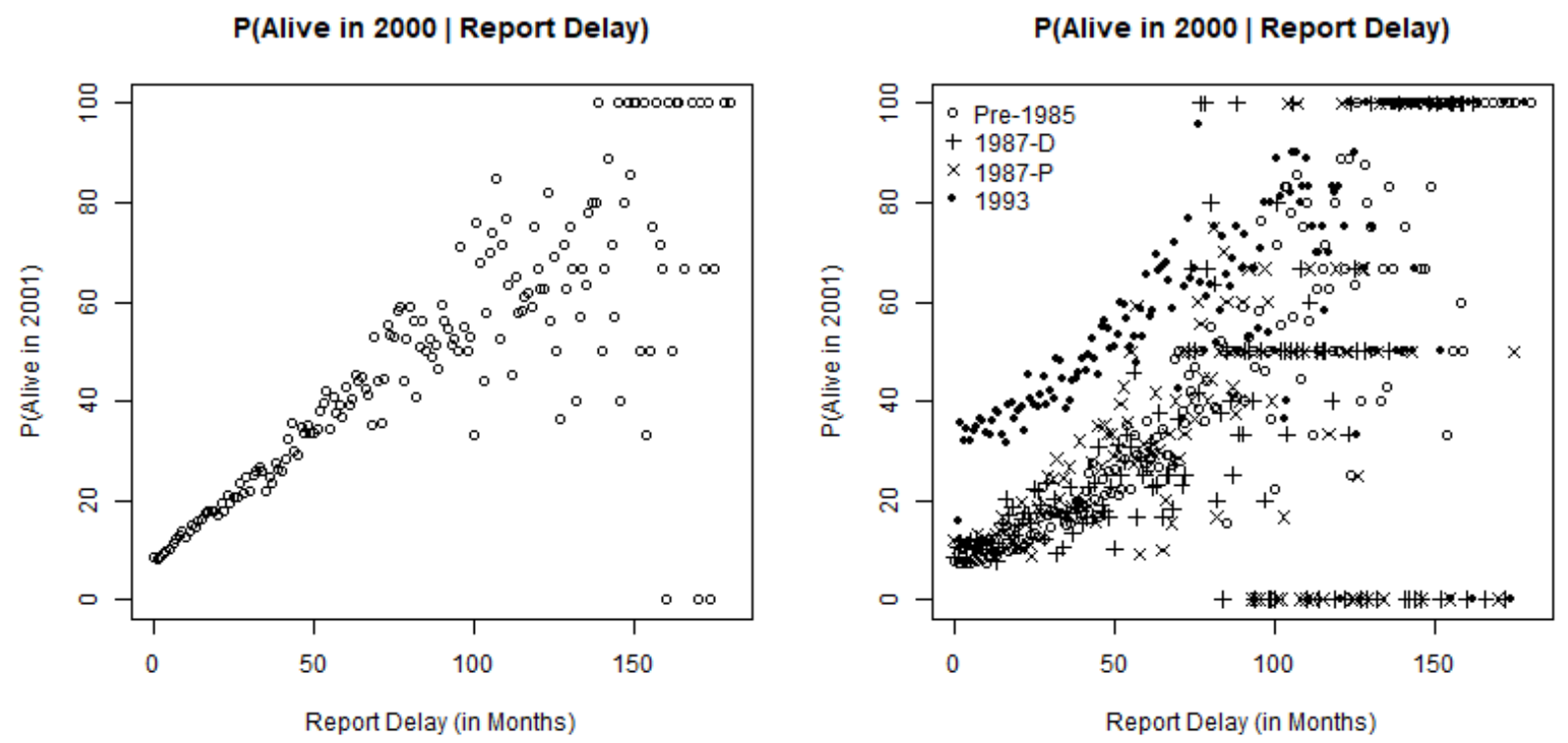

Note: Figure represents mortality results by report delay. The left panel combines all diagnosis definitions together, whereas the right panel splits them up.

include the 1993 severe HIV-related immunosuppression surveillance definition to highlight the leniency of this new definition compared to the older definitions.

Figure A.8 the empirical CDF of report delay. While there are some extremely large report delays, about $60 \%$ are within 6 months. Figure A.8 displays the average report delay by month and surveillance definition. Clearly, there are extreme report delays for each surveillance definition before it is put into use. Second, there appear to be large delays in each June.

\section{A.8 Additional Identification Figures}

Figure A.11 displays the percent of respondents indicating having obtained information about HIV/AIDS from radio, television and/or newspapers sources. We observe a large and statistically significant increase in each category immediately following the week Johnson announced his diagnosis. This is an important finding that shows how the public became more aware of HIV/AIDS.

Figure A.12 displays the number of mentions of Magic Johnson's name in newpapers around the United States. These data come from newspapers.com, a subsidiary of Ancestry.com. There is a clear spike in the mentions of Johnson, even more so than previous peaks which coincide with Johnson's NBA playoff success. 
Figure A.8: Empirical CDF of Report Delay

\section{Empirical CDF of Report Delay}

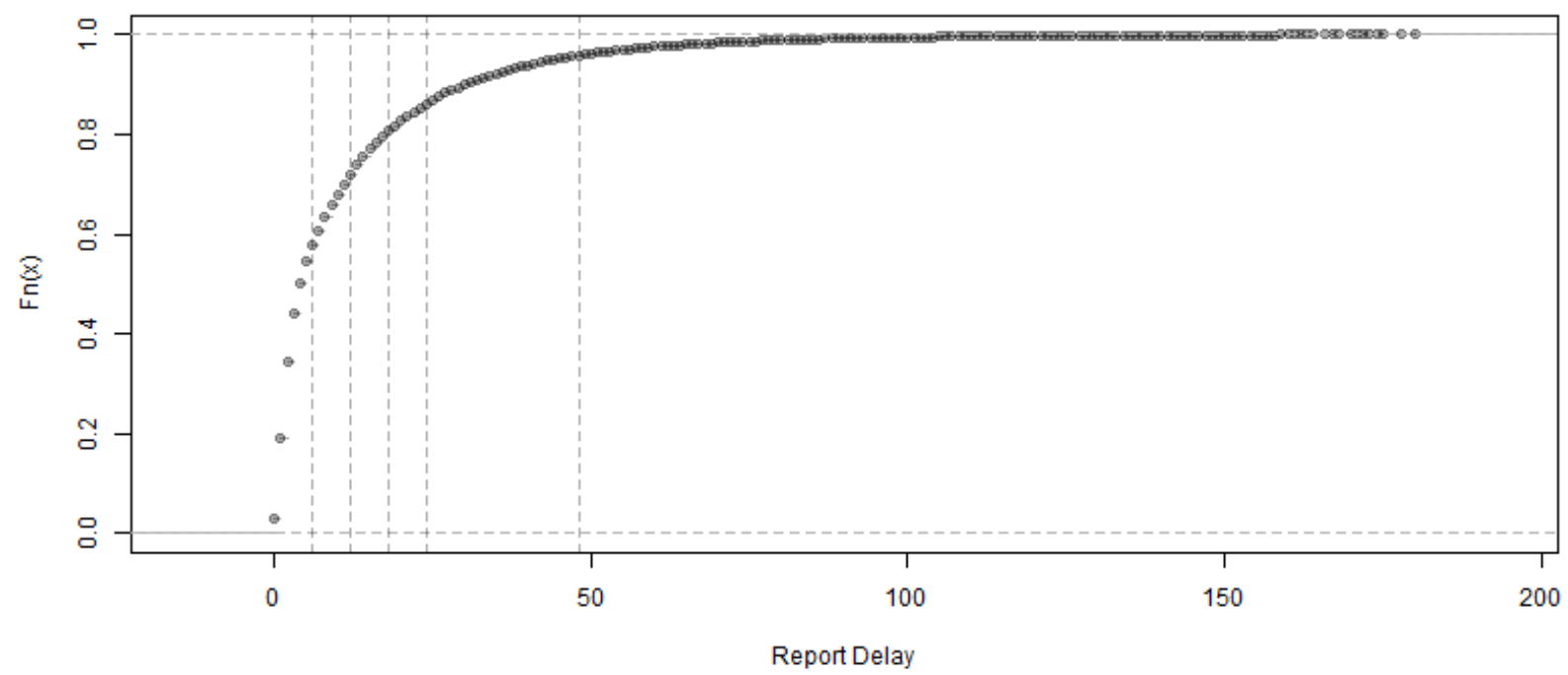

Note: This figure represents the empirical cumulative density function of report delay. The vertical lines depict $6,12,18,24$ and 48 months out from a diagnosis.

Figure A.9: Average Delay in Reporting by Definition and Diagnosis Month

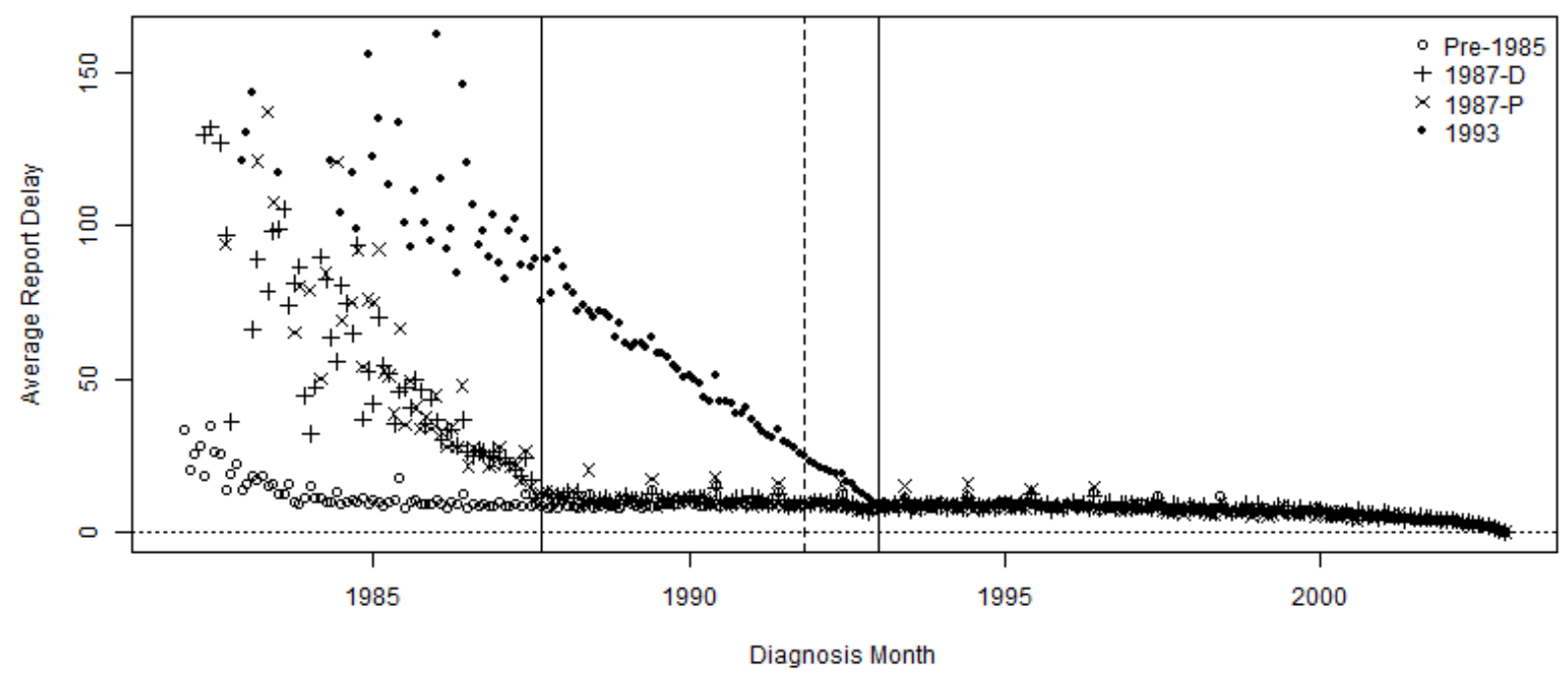

Note: This figure depicts the average report delay by diagnosis month and surveillance definition. The vertical lines represent the beginning, end, and date of Johnson's announcement. 
Figure A.10: Dec. 7, 1991 NYT Front Page Article

\section{H.I.V. Tests Up 60\% \\ Since the Disclosure \\ FromMagic Johnson}

\section{By CALVIN SIMS}

The number of people being tested for H.I.V. has increased almost 60 percent in New York City and has risen sharply throughout the nation in the month since Magic Johnson announced that he was infected with the virus that causes AIDS.

In New York, the increase has strained the resources of the City Health Department, which said it can now take up to seven weeks to get an appointment for a test at the city's counseling and testing centers. Before Mr. Johnson's announcement on Nov. 7 , most centers provided tests without an appointment or had a maximum wait of two weeks.

Officials of the Federal Centers for Disease Control in Atlanta said yesterday that while no statistics had been compiled, a random survey of state health departments nationwide showed an equally sharp increase in the number of people seeking tests for H.I.V.

“It's all anecdotal at this point, but in some cities we are hearing of increases as large as 10 times the number of tests previously requested," said Fred Kroger, director of AIDS education and information programs for the centers. Mr. Kroger attributed the rise in H.I.V. testing to people who are not now engaged in risky sexual behavior, but are

Note: Excerpt from the New York Times front page on December 7, 1991. This, in addition to Cohn et al. (1992), provide strong anecdotal evidence of Johnson's effect on testing. 
Figure A.11: Past Month Sources of AIDS Information in 1991
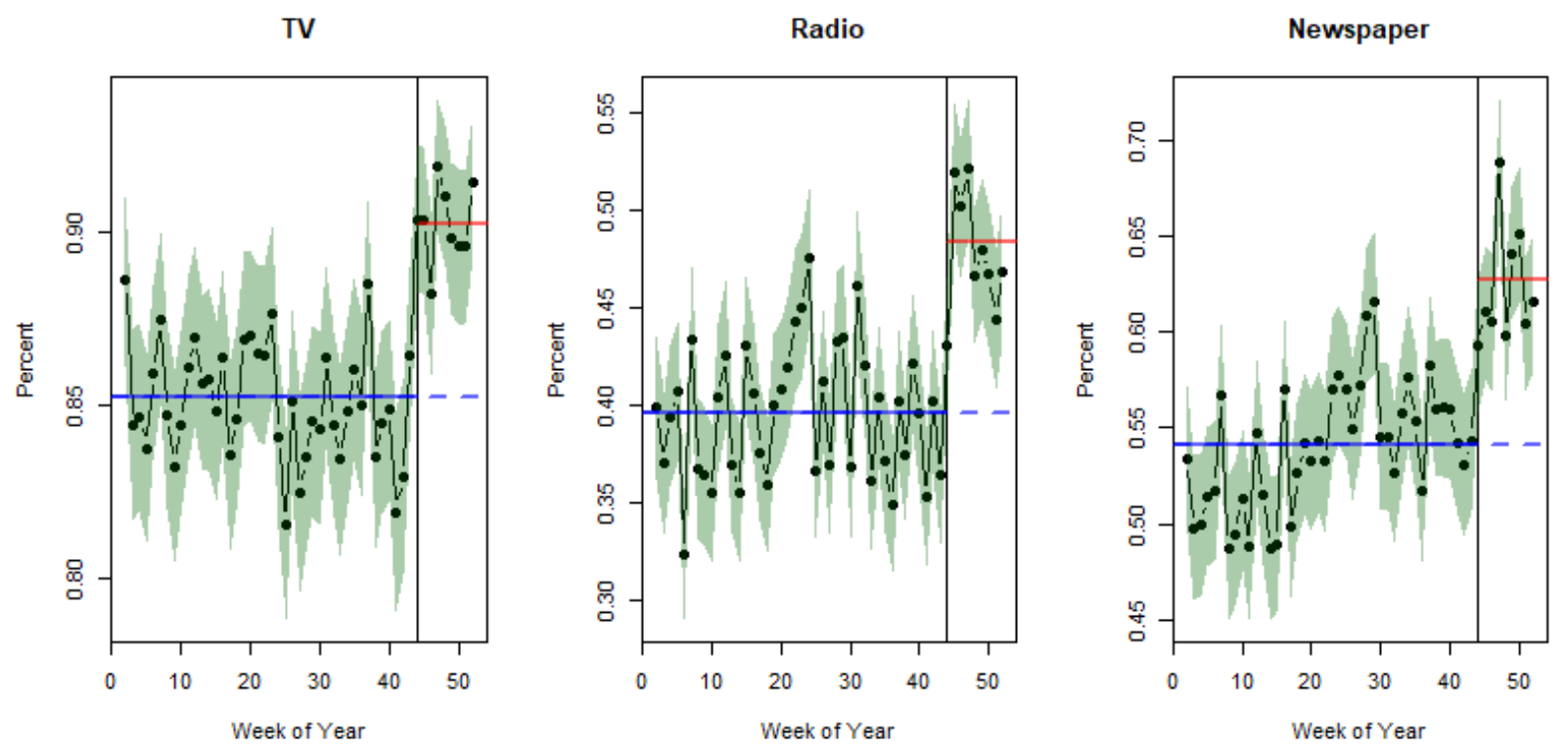

Note: This figure shows the percent of individuals who had heard about HIV/AIDS via TV, Radio or Newspaper media during 1991. The large spikes coincide with the week of Johnson's announcement.

Figure A.12: Daily Newspaper Mentions

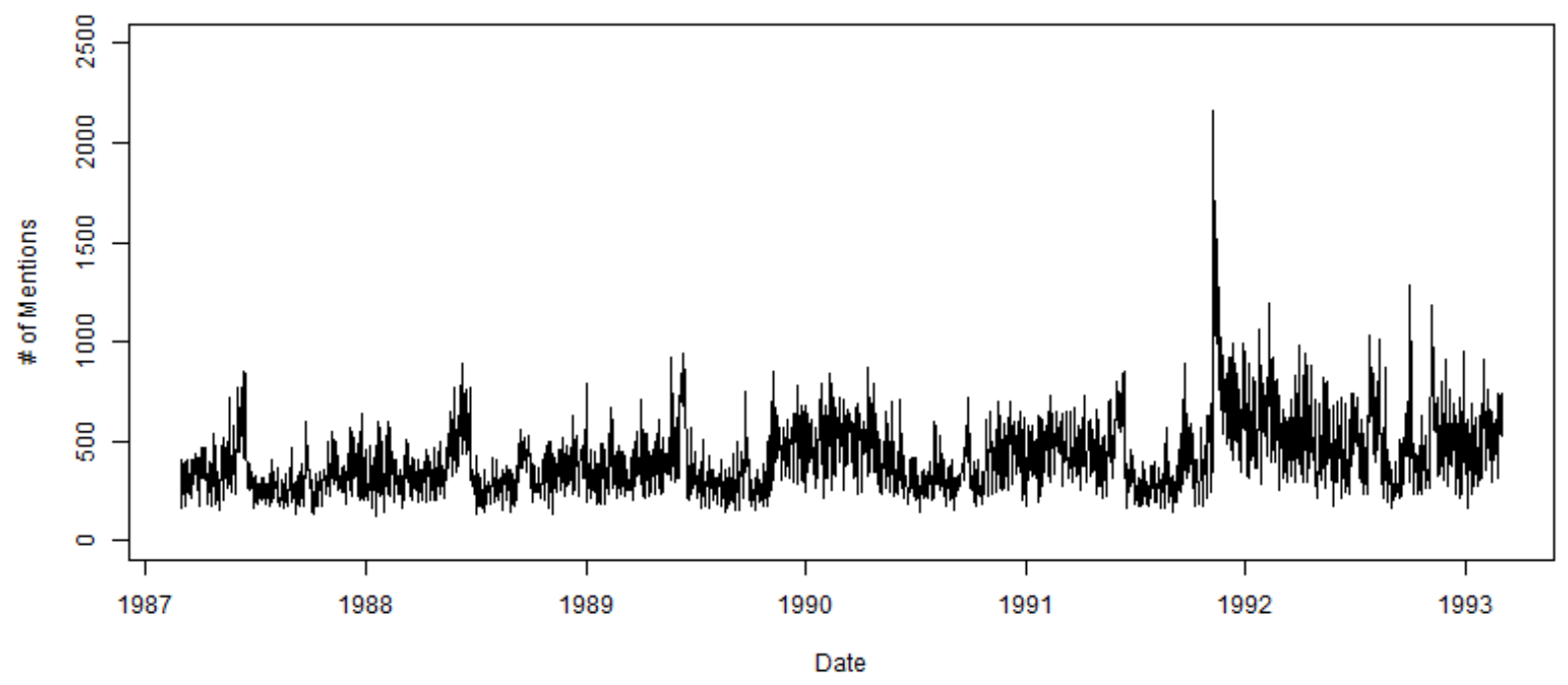

Note: Figure depicts the number of times "Magic Johnson" appears in newspapers across the country by day. This spike occurred on November $7^{\text {th }}, 1991$, the day Johnson announced his positive HIV status. These data comes from newspapers.com's API, a subsidiary of Ancestry.com 